\title{
Analysis of Accelerometer Data from a Woven Inflatable Creep Burst Test
}

\author{
George H. James ${ }^{1}$, Michael Grygier ${ }^{1}$, and Molly M. Selig ${ }^{2}$ \\ NASA Johnson Space Center, Houston, TX 77058
}

\begin{abstract}
Accelerometers were used to montor an inflatable test article during a creep test to failure. The test article experienced impulse events that were classified based on the response of the sensors and their time-dependent manifestation. These impulse events required specialized techniques to process the structural dynamics data. However, certain phenomena were defined as worthy of additional study. An assessment of one phenomena (a frequency near $1000 \mathrm{~Hz}$ ) showed a time dependent frequency and an amplitude that increased significantly near the end of the test. Hence, these observations are expected to drive future understanding of and utility in inflatable space structures.
\end{abstract}

\section{Nomenclature}

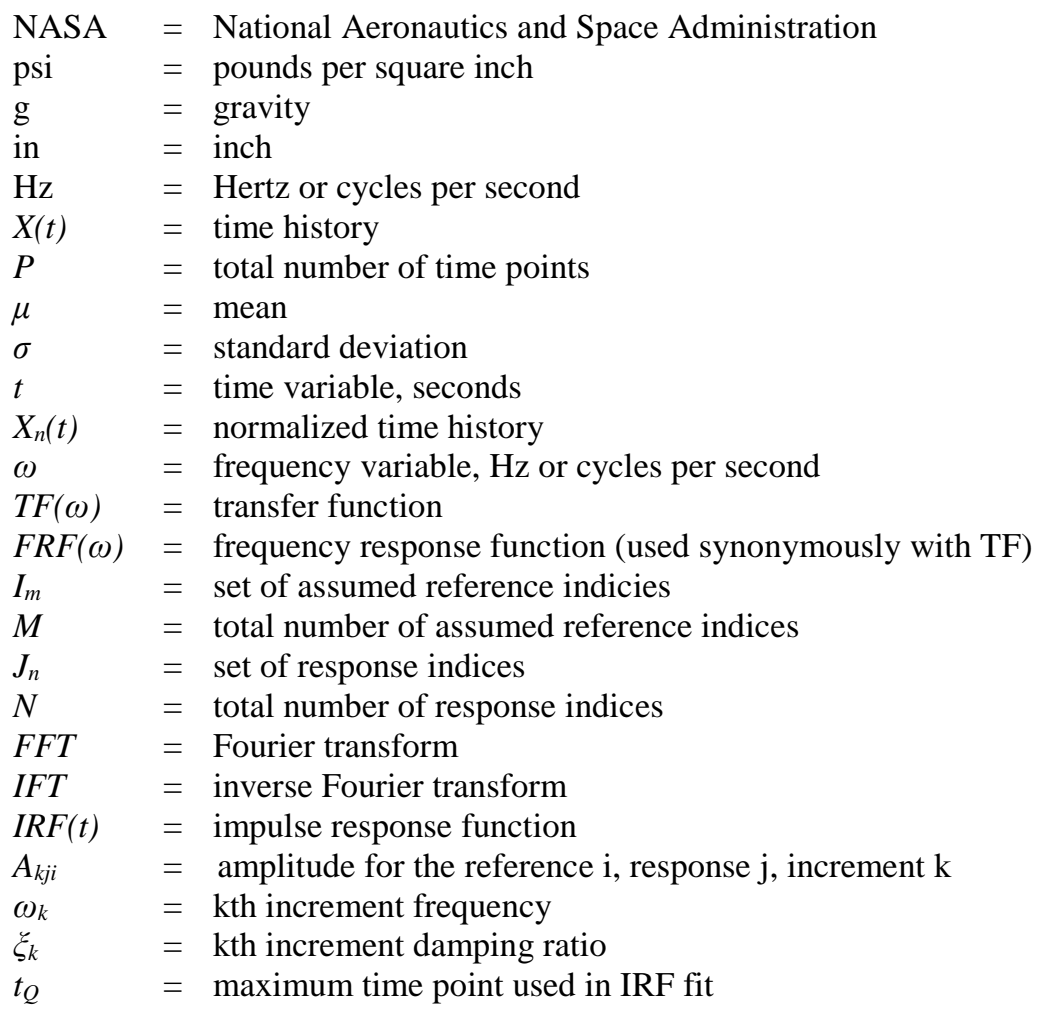

\footnotetext{
${ }^{1}$ Senior Aerospace Engineer, Loads and Structural Dynamics Branch, ES6, AIAA member

${ }^{2}$ Aerospace Engineer, Structure Branch, ES2, AIAA Member
}

American Institute of Aeronautics and Astronautics 


\section{Introduction}

$\mathrm{T}$ he structural dynamics response of any structure (even an inflatable one) represents measureable metrics that may be interpreted for understanding of structural health, environmental loading, induced loads, test margins, or analytical fidelity. These responses show up on a continuum of global response (generally low frequencies) to highly localized responses (generally higher frequencies). Accelerometers are the most common and versatile sensors for structural dynamics measurements. Typically, structural dynamics measurements are processed with a known measurement of the forces being imposed on the structure. Alternatively, operational analysis techniques are used when no distinct measurement of the input force is available ${ }^{1}$.

This paper provides the first phase of data analysis from the accelerometer complement of a creep burst test on an inflatable test article. This NASA Johnson Space Center test article was a woven Vectran inflatable module 88 inches in diameter and 10 feet long and was tested until failure from creep. The test article was pressurized to an internal pressure of $145 \mathrm{psi}$, and was held at pressure until burst. The external environment remained at standard atmospheric temperature and pressure. The module burst occurred after approximately four hours of slow pressure increases and 49 minutes at the target pressure. In addition to the accelerometers mentioned above, the test article was also instrumented to monitor pressure, temperature, sound, and video. Additionally, photogrammetry was used to obtain strain measurements of a limited area test patch. This paper is a companion to the second reference that discusses the test article, test setup, predictions, observations, photogrammetry strain results, and a comparison of the module level creep behavior to the strap level creep behavior ${ }^{2}$.

Figure 1 shows images of the test article in the test chamber with instrumentation attached. The green metallic section is an endplate. There is an endplate on the opposite end of the article. These endplates are connected with a solid circular axle. The end of the axle protruded from the endplates and is shown in Figure 1 as supported externally by a blue test frame. The tan Vectran overwrap is seen, which carries the inflation loads of an interior bladder (which cannot be seen). The round black buttons are used to stabilize the internal bladder to the overwrap. The accelerometers and attached cabling can be seen as well as the white photogrammetry patch. For more information on the test article, see the second reference.
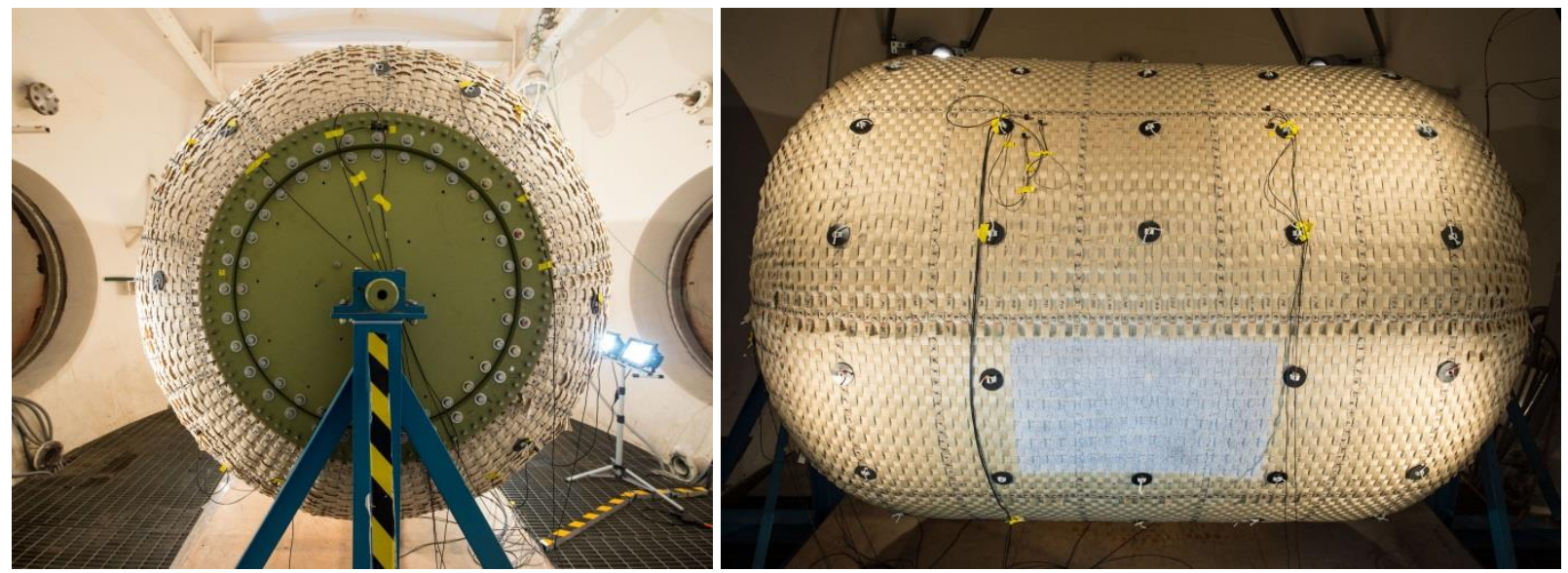

Figure 1. The Creep Burst Test Article. The module is shown inside the test chamber.

\section{Structural Dynamics Data}

Fifteen Kistler 8632C10 PiezoBEAM cube accelerometers were mounted on the test article while inflated at 2 psi delta pressure. These piezoelectric sensors were rated to measure $10 \mathrm{~g}$ of acceleration in either direction and were configured as a cube with a .56-in x .56-in x .56-in footprint. Previously, these sensors were used for structural health monitoring measurements on the Space Shuttle Orbiters as part of the Shuttle Modal Inspection System (SMIS) ${ }^{3}$. The sensors did not survive the failure of the test article. Figure 1 shows global context images of the sensors and the associated cabling installed on the test article. Figure 2 shows close-up images of sensors installed on the soft goods and metallic core of the test article. Figure 3 and Figure 4 provide schematics of the test article with the accelerometer locations and directions denoted.

American Institute of Aeronautics and Astronautics 

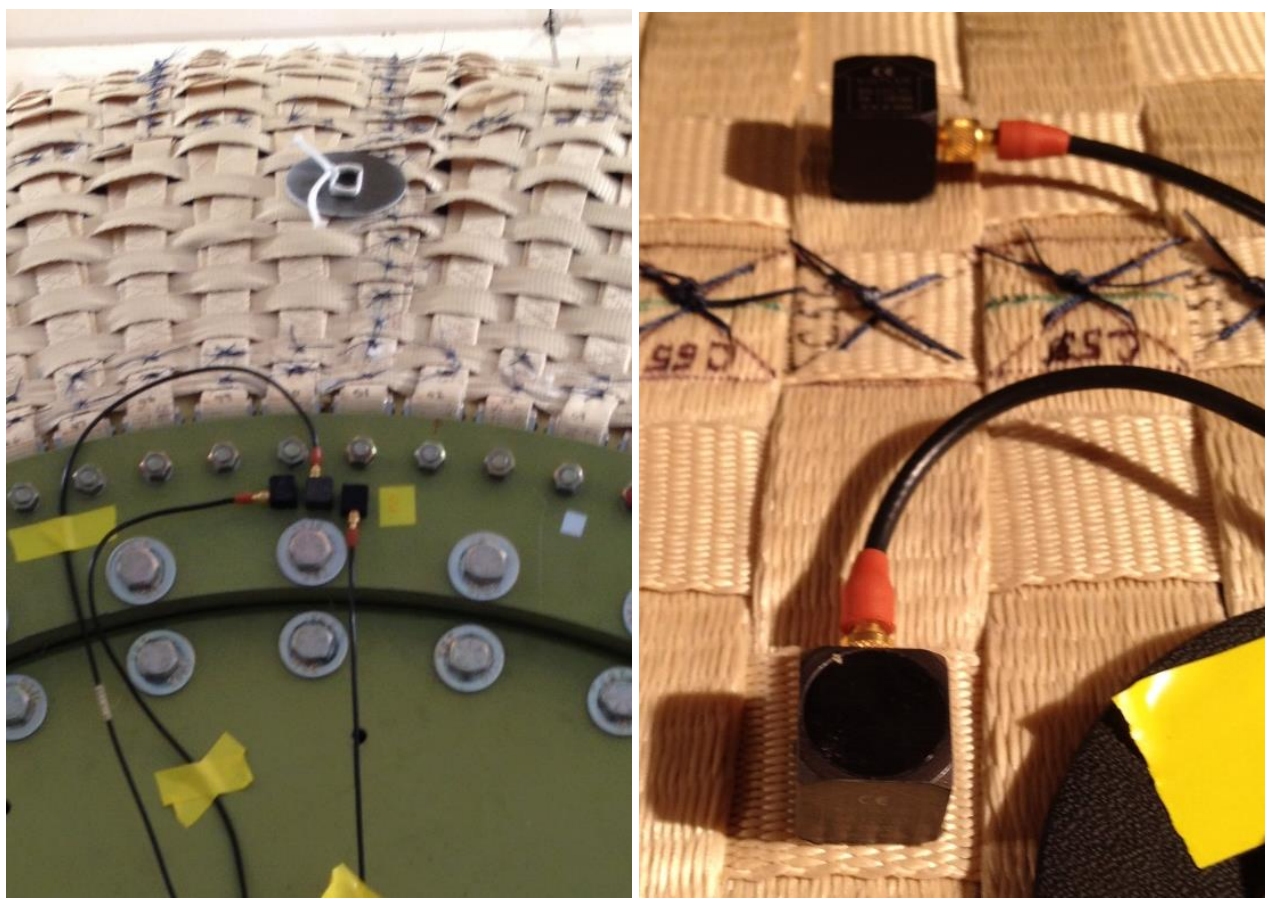

Figure 2. Close-up Images of Installed Accelerometers on the Creep Burst Test Article
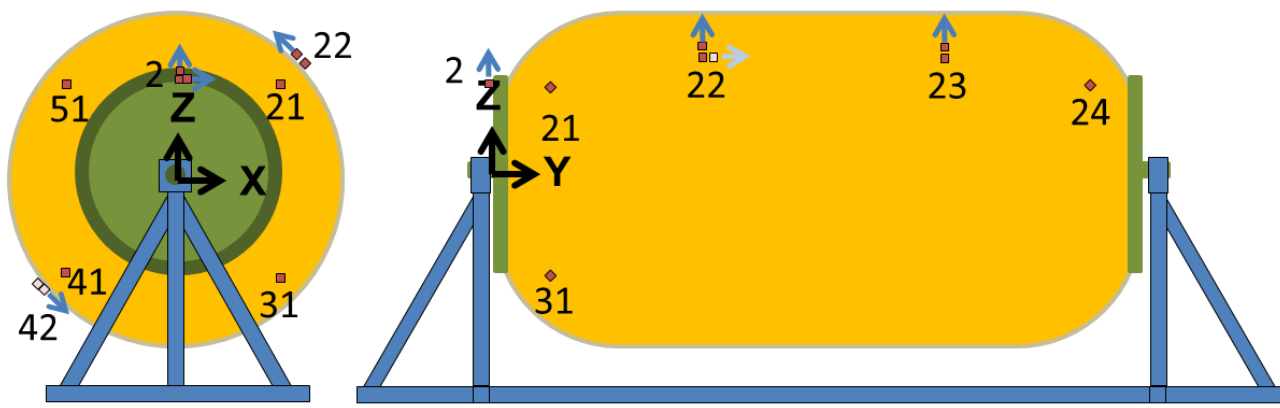

Side View

Front View

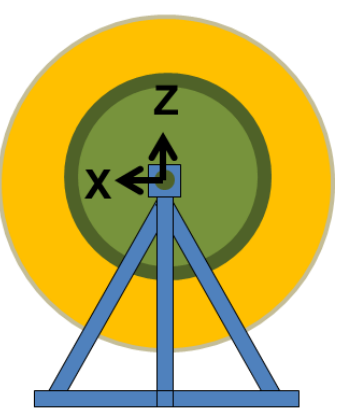

Side View

Figure 3. Location and Direction Schematic of Installed Accelerometers (Three Views)

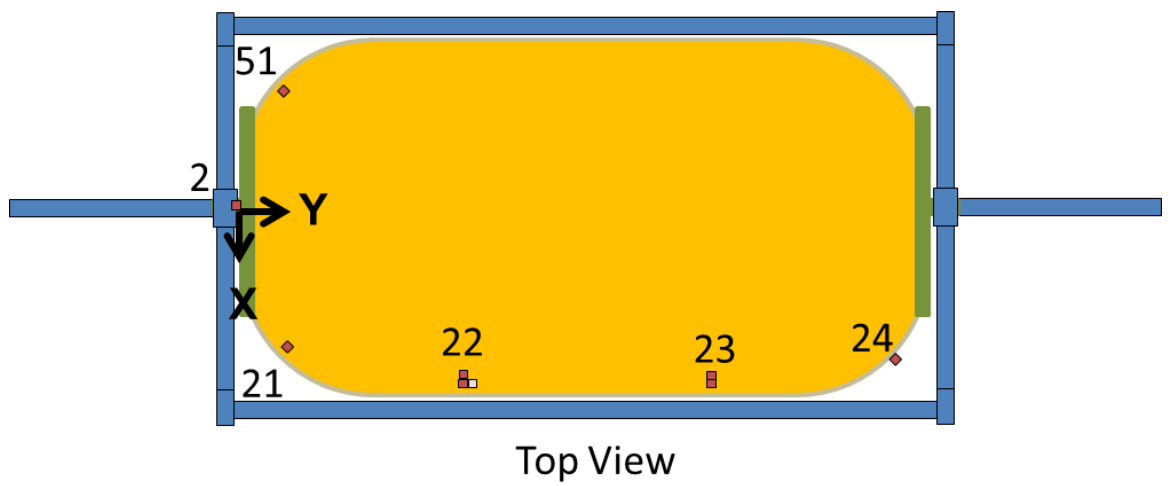

Figure 4. Location and Direction Schematic of Installed Accelerometers (Top View) 
An important consideration in preparing for the test was how to attach the accelerometers to the soft-goods of the test article. Operationally, the sensors needed to be installed at the low test article pressure of 2 psig, yet needed to remain attached and function up to and including the final test article pressure of $146 \mathrm{psig}$. It was anticipated that the straps of the restraint layer would expand and move during the ramp up to the test pressure. Also, the mounting technique had to be robust to function for several weeks or months (if the test had extended that long) as well as provide no-impact to the performance of the test. Another consideration was that it was expected to be difficult to provide any resistance force to the sensor during mounting except for the 2 psig internal pressure of the test article.

To develop a mounting technique, a study was performed using a single strap of the restraint layer material loaded in a tensile test machine. Two different adhesive tapes (which had previously been tested for other long-term sensor installation applications) were assessed. Each adhesive was assessed using an installation with and without back pressure being applied during installation. The single strap was loaded with the equivalent force that a similar strap would see on the test article with an internal pressure of 2 psig. Four sensors were installed in each of these test case combinations and then the tension was increased to the equivalent of the 146 psig test pressure. Figure 5 shows this test. Both adhesive systems performed well during the test both with and without back pressure. However, the $3 \mathrm{M}$ F9473PC adhesive tape appeared to be a more tenacious application with no back pressure and was selected for use to mount the accelerometers in the test.

During the actual creep burst test, 15 accelerometers were mounted on the test article and 12 of these were monitored. A Bruel and Kjaer LAN-XI Type 3053-B-120 portable data acquisition module driven by the B\&K Pulse software was used for data acquisition. Using Figure 3 and Figure 4 as references, the two sensors at location 42 and the $\mathrm{Y}$ direction sensor at location 22 were not monitored. These sensors were to be treated as spares/supplements for use during the test, which could have lasted several weeks. Microdot to BNC cables of 120 -foot length were used to connect the sensors to the 3053 module, which was in a shielded control room adjacent to the chambered test article. It should be noted that these sensors, the 3053 module, and the Pulse software were used to perform a traditional hammer-based modal test on the test article prior to installation in the chamber as well as during the single strap test mentioned above.

The data acquisition was set up to take data in contiguous five minute blocks of data, which were sampled at 4096 samples per second. Anti-aliasing filtering was used and the resulting data have been assumed useful up to $1300 \mathrm{~Hz}$. A nearly continuous five-hour data stream from test start until failure was recorded. The test article was pressurized from .5 psig until 146 psig with specific pressure holds at 5, 15, 36, 50, 75, 100, and 125 psig to allow five-minute increments of steady state accelerometer data before reaching the final test pressure of $146 \mathrm{psig}$. Figure 7 provides both a full five-minute data sample, and the first one-second data sample, at location 21 (refer to Figure 3 and Figure 4) during the low-pressure 5 psig hold. The data appear as would be expected with an identifiable vibration signature. Figure 8 also provides both a five-minute, and the first one-second data sample of the sensor at location 21 at the final test pressure of $146 \mathrm{psig}$. Note that the dominant features are now randomly-spaced, impulse excitation events. These events not only visibly dominate the plots but also can significantly raise the vibration floor of the data when they occur. A detailed assessment of these impulse events will be covered in a later section of this paper.

\section{Data Assessments}

There are three basic data analyses that will be discussed in the following sections. The first will look at the impulse events that dominate the response throughout the test and most of the pressure ramp-up phase ${ }^{2}$. The intent will be to detail observational information that can inform the discussion of the source and life-effects of these impulses. The next assessment discussed will be an assessment of the general modal content of the test article over the life of the test. Although there are limitations in the ability to extract traditional modes in the presence of the impulse events, techniques have been used that do enhance the data return. This global modal assessment provides a view of the primary dynamics content of the system and suggests where detailed assessments would be advantageous. The final assessment that will be discussed is one such detailed extraction and tracking of a dynamic phenomenon near $1000 \mathrm{~Hz}$. This phenomena becomes very dominant near the end of the test and has some interesting trends. A final section will address the planned data assessments that will follow the work presented herein.

American Institute of Aeronautics and Astronautics 

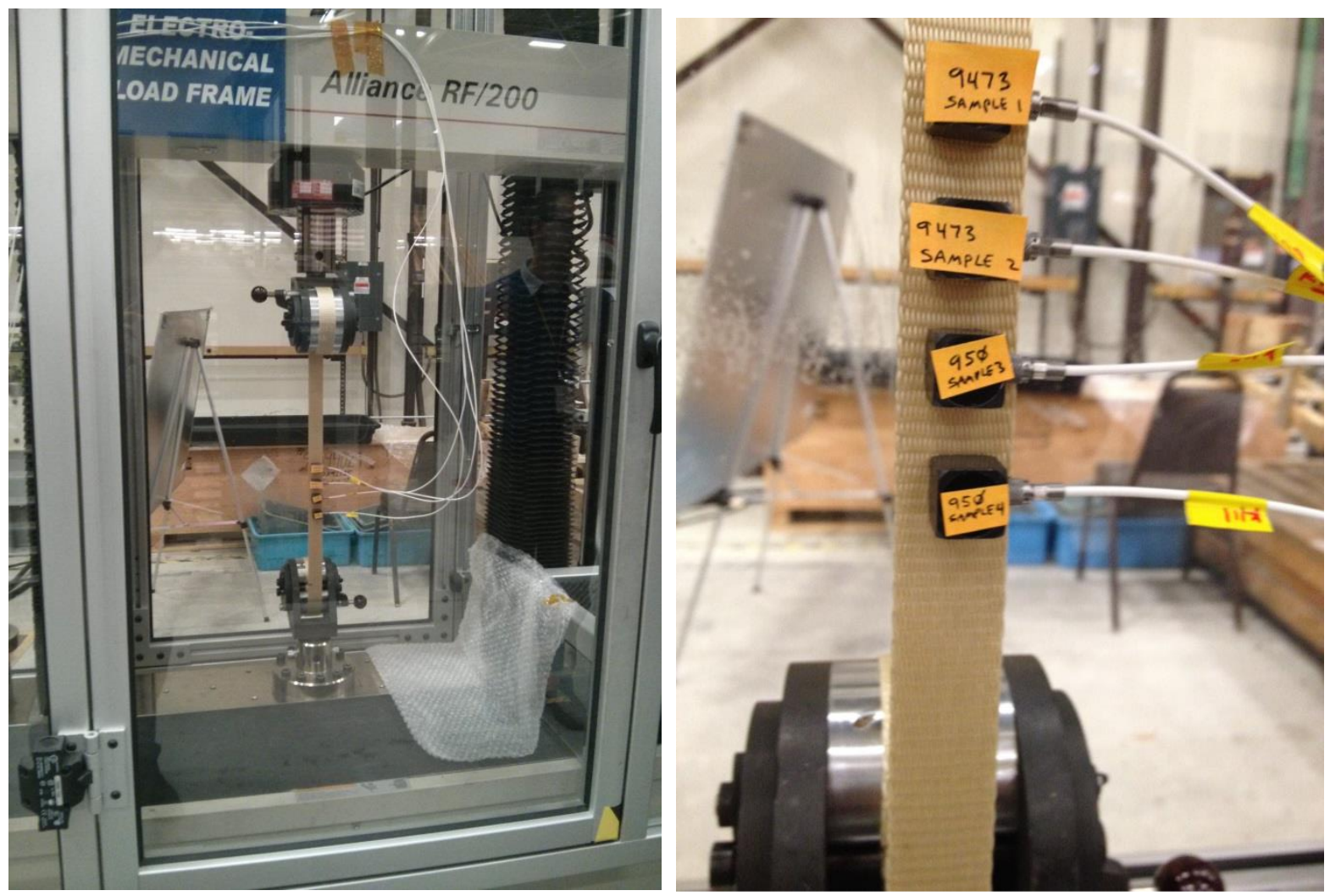

Figure 5. The Single Strap Test to Assess Adhesive Accelerometer Mounting Systems

\section{Impulse Events Assessment}

\section{Standard Deviation Metric}

The ambient induced vibration monitored by the 12 sensors varied significantly from sensor to sensor and over the course of the test. Therefore in order to allow a common base of comparison, the standard deviation was estimated for each sensor trace assessed. Assume that $X(t)$ is an acceleration time history from a data increment with $N$ individual time measurements, each of which is denoted as $x\left(t_{i}\right)$. The mean value is defined as

$$
\mu=\frac{1}{P} \sum_{i=1}^{P} x\left(t_{i}\right)
$$

The standard deviation or sigma is then determined via the following:

$$
\sigma=\sqrt{\frac{1}{P} \sum_{i=1}^{P}\left[x\left(t_{i}\right)-\mu\right]^{2}}
$$

The raw response of each sensor $X(t)$ was then normalized by that standard deviation:

$$
X_{n}(t)=\frac{X(t)}{\sigma}
$$

In that manner, much of the following data assessments were performed and reported with nondimensional units of "number of standard deviations". Note that these standard deviation values are a metric of how excited that sensor is in the data increment under study as well as a measure of the "typical" ambient response amplitude of the structure. The impulse events of interest in the section are then studied as deviations from the normalized ambient response.

American Institute of Aeronautics and Astronautics 

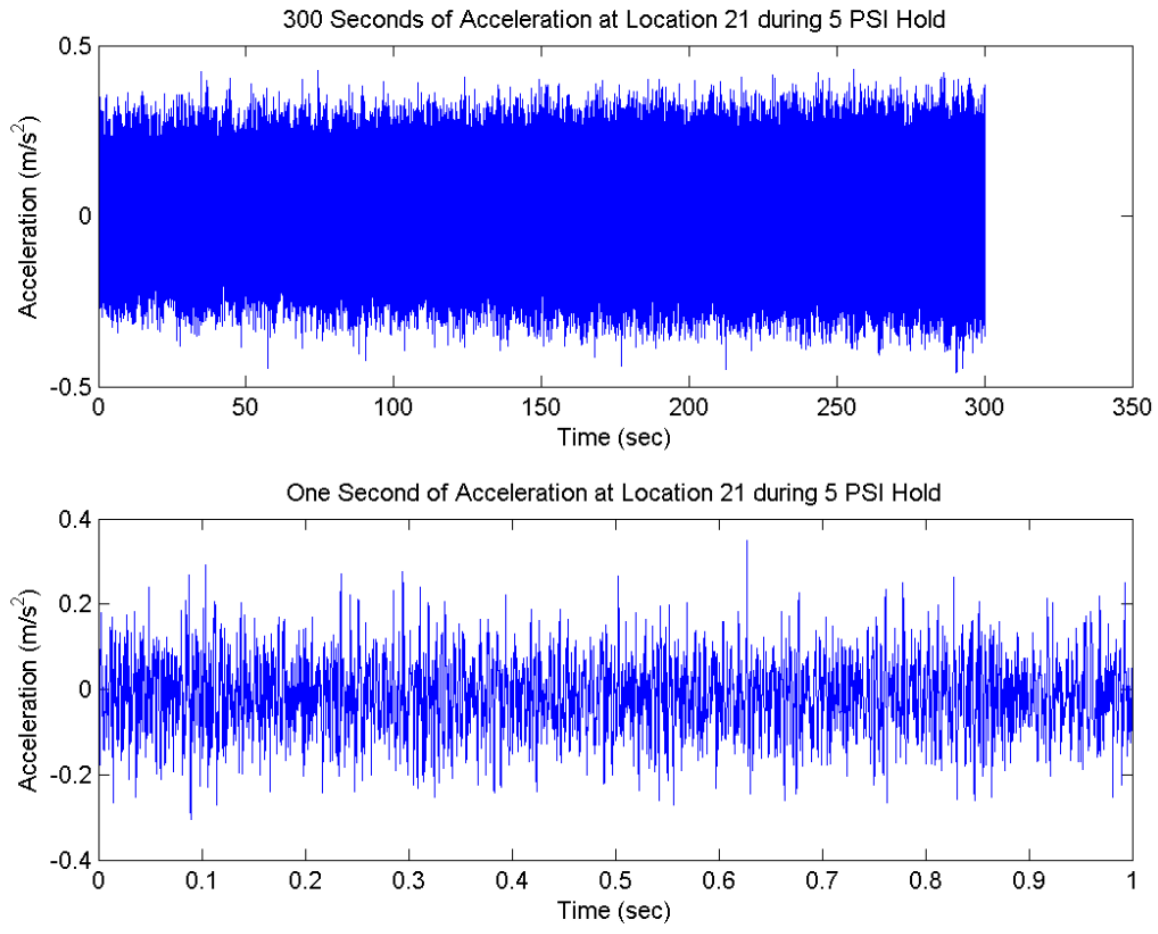

Figure 7. Five Minute and One second Accelerometer Time History at 5 PSI Hold
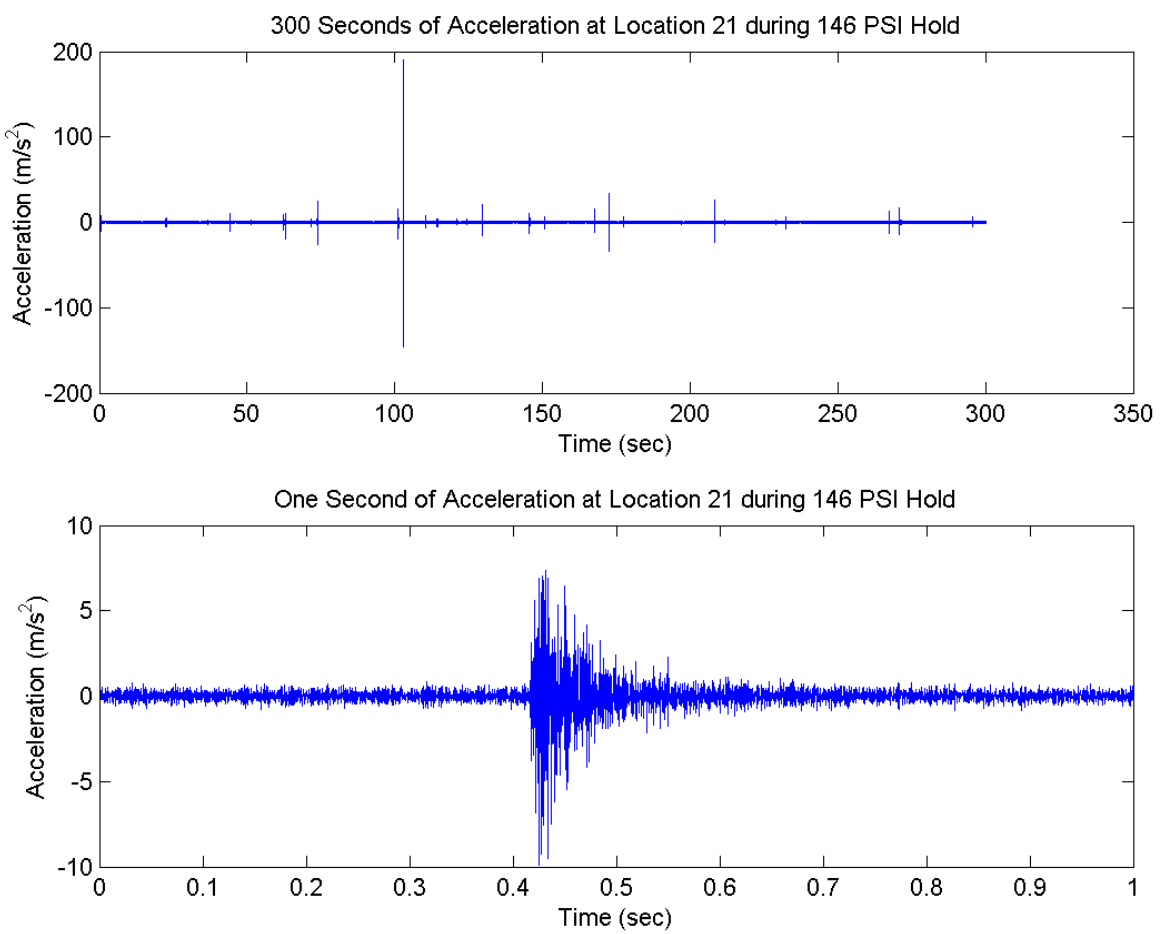

Figure 8. Five Minute and One second Accelerometer Time History at 146 PSI Hold 
Figure 9 shows the standard deviation (or sigma) for sensor 51Y. Time is the independent variable in the top plots and pressure is the independent variable in the lower plots. In these plots, the standard deviation for each data increment is denoted as an individual "*" symbol or one of the following alternatives. Some events were pressure holds (as opposed to pressure ramps) and are denoted by "+" symbol. Some data increments did not contain the full 5 minutes of data and are noted with the "o" symbol. A trace line connects these measurement only to aid in visualization. The left hand plots provide the original data with outlier peaks due to sensor saturation. In sensor saturation, the accelerometer experienced a high frequency internal resonance and temporarily ceased to provide meaningful data. Hence, a saturation event is an indication that there is a unique high frequency content in a specific impulse event. The sensor saturation effects due to a high frequency component may be filtered out by the anti-aliasing filter but the physical effects on the internal resonance still occurs. As a note, the internal resonance of the sensors was $22,000 \mathrm{~Hz}$. A sensor saturation event is clearly indicated as the sigma value drastically and increases. The right hand plots show the sigma values with saturated peaks replaced by assumed values taken from the last non-saturated data increment. These unsaturated and assumed values were used for normalization.

Overall there appears to be a trend of increasing standard deviation as the pressure increases from 15 psi to near max pressure. This excitation seems to plateau at its highest levels during the variable pressure segments. The standard deviation appears to seek a lower level during pressure hold increments. The excitation level is likely a combination of environmental effects (gas flow; pumps, valves, fans, road noise) and the impulse events.

Figure 10 provides another assessment of the standard deviation as a metric for excitation level. The standard deviation amplitude vs. the total number of impulse events is shown. The top plot shows the assumed standard deviation vs. the total number of events (migration plus extinction). The middle plot expands and scales that comparison by adding that total number with the saturation events scaled up by a multiplier of 10 . This multiplier was chosen as the general increase in the value of the standard deviation is an order of magnitude. The bottom plot compares the original standard deviation to the scaled number of impulses. The same plots have been generated for all 12 sensors but are not reported here. Plots such as Figure 10 are to assist in determining if there is a relationship between excitation levels and the impulse events. Additional efforts to study of these trends might entail:

a. adding another significant digit to the sigma values;

b. relating impulse events within an individual sensor instead of the total number of events across all sensors; or

c. determination of higher fidelity standard deviation values from the non-saturated parts of each sensor time history instead of assuming a value from earlier data segments.

\section{Categorization of Impulse Events}

The processed data (including the pressure ramp-up and the hold data) suggested four categories of impulse events. The first class are labeled as "migration" events and are greater than 10 standard deviations (or sigma for short). Some impulse events were uncharacteristically larger in amplitude. These were collected in a separate family called "extinction" events and were greater than 500 sigma. Some of these impulse events (either migration of extinction) had the effect of producing single sensor saturation and are listed as "saturation" events. There were a few extinctionclass events that actually saturated all sensors on the test article. These were categorized as "global" events.

Figure 11 is an example of a results plot for an individual sensor showing this characterization. This example includes the data from sensor 21 in the Y direction from a data increment at 146 psi. The estimated standard deviation is in the title of the upper left hand plot. The analysis uses an assumed standard deviation as saturation events add bias offsets to the standard deviation estimates. Since these plots provide the data in amplitudes of number of standard deviations, an assumed standard deviation is used to normalize the data under assessment. The title of the upper left hand plot contains this assumed standard deviation and the assumed standard deviation is in the upper right hand plot title.

The high amplitude impulse events (extinction events) are denoted using the circles and the moderate amplitude events (migration events) are shown as asterisks. For this data analyses, the cut-off for migration events was 10 standard deviations (denoted by the lower cut-off) as seen in any sensor. The upper cut-off for extinction events was 500 standard deviations as seen in any one sensor. The upper left hand plot shows the semilog plot of the absolute values. The migration and extinction cut-offs are shown. Note that an impulse event may be defined in another sensor and be below the appropriate cut-off on these plots. The upper right hand plot shows the linear plot of the data with positive and negative impulse values denoted. The lower left plot shows only the absolute values of the extinction and migration events without the rest of the sensor data. The lower right hand plot only shows the lower level Migration events. Figure 12 shows a data set that more readily illustrates the sensor saturation phenomena after the two extinction peaks. This data is from sensor at location 23 in the Y direction from a data set at 146 psi.

American Institute of Aeronautics and Astronautics 

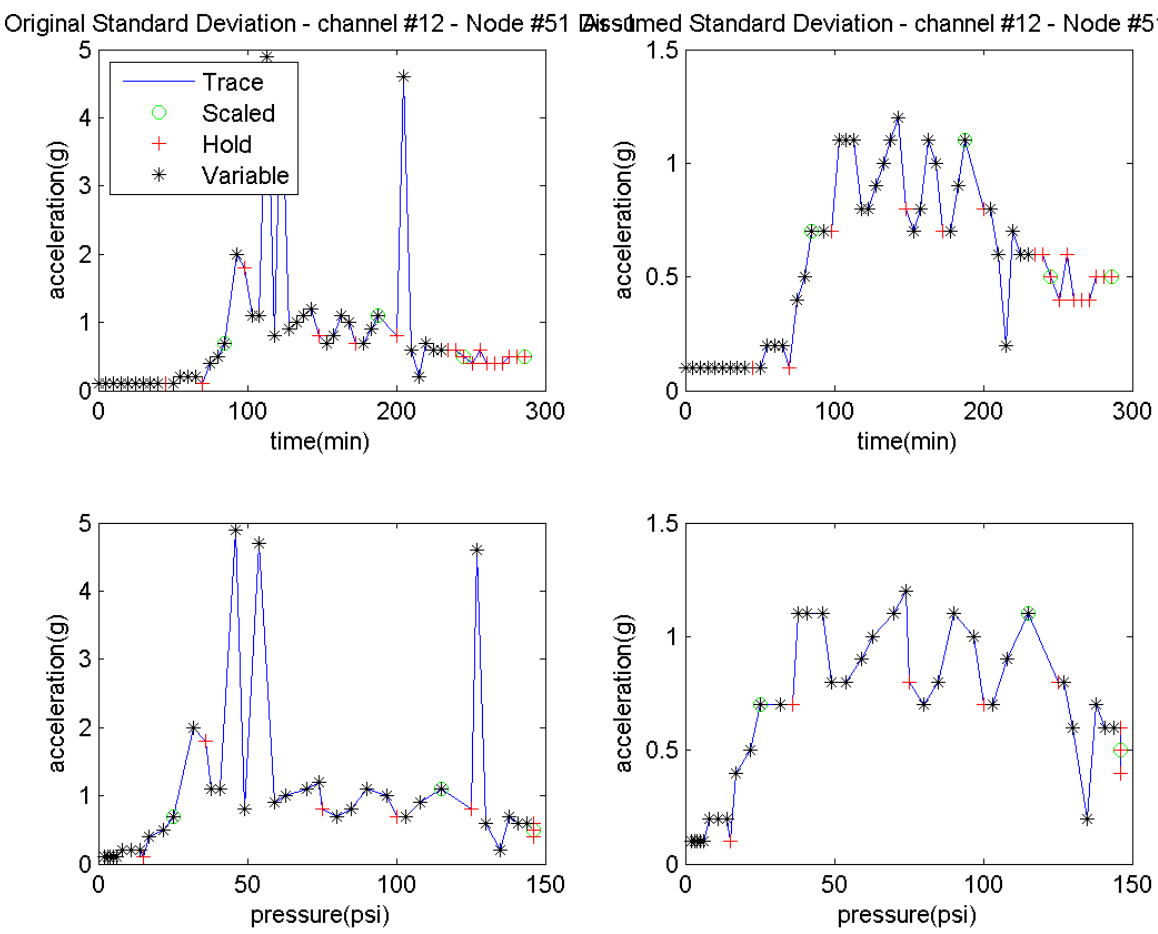

Figure 9. Original and Assumed Standard Deviation vs. Time and Pressure for Sensor 51Y
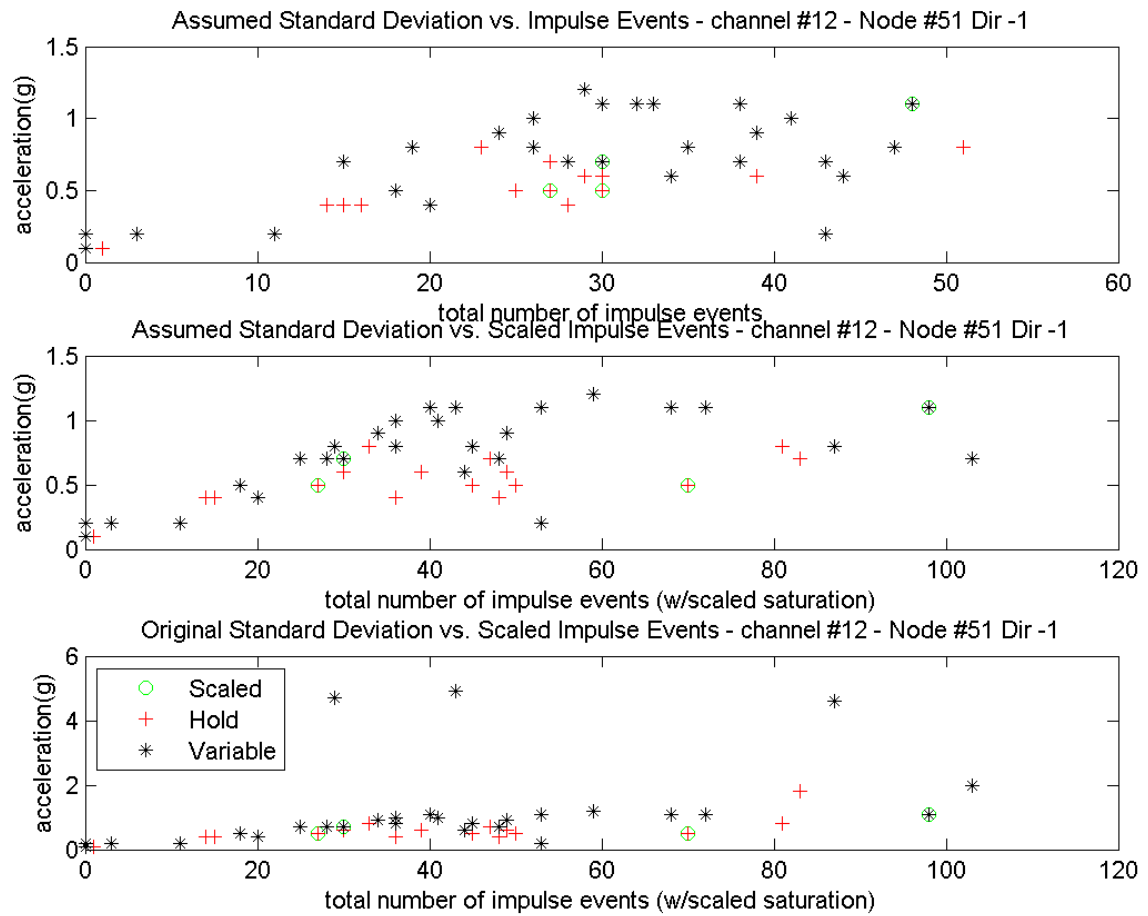

Figure 10. Original and Assumed Standard Deviation vs. Number of Impulse Events for Sensor 51Y

American Institute of Aeronautics and Astronautics 

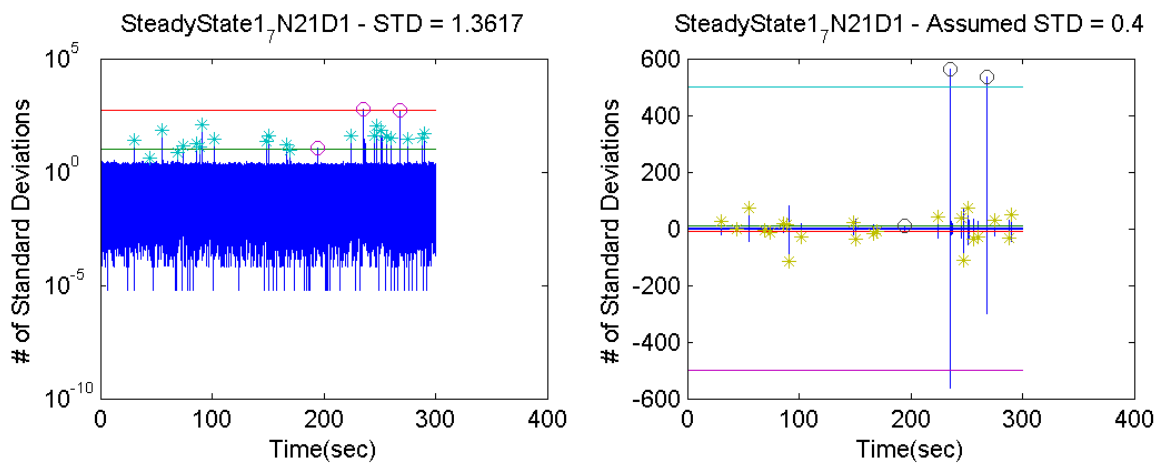

SteadyState ${ }_{7}$ N21D1 - extinction and migration level pops SteadyState ${ }_{7}$ N21D1 - migration level pops
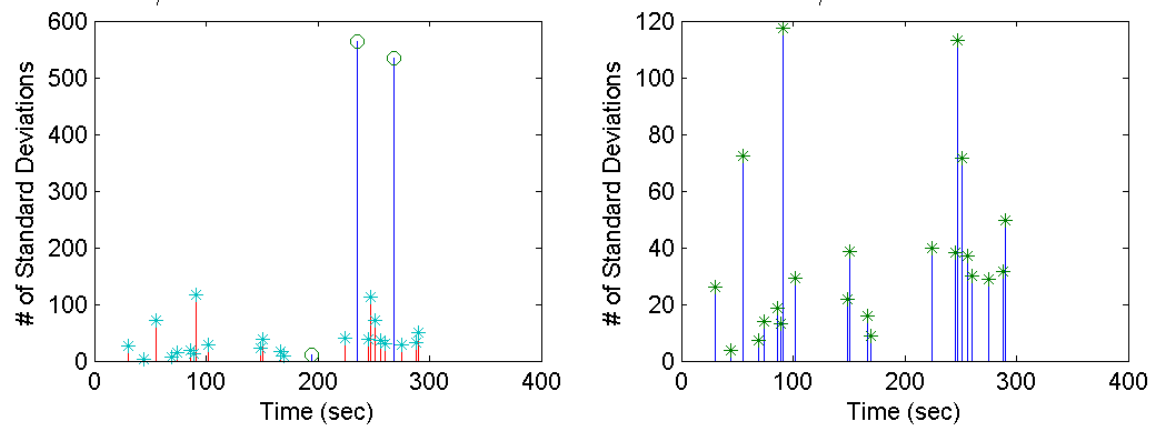

Figure 11. Example Strap Pop Analyses Plots for 21Y from a Data Increment at 146 psi.
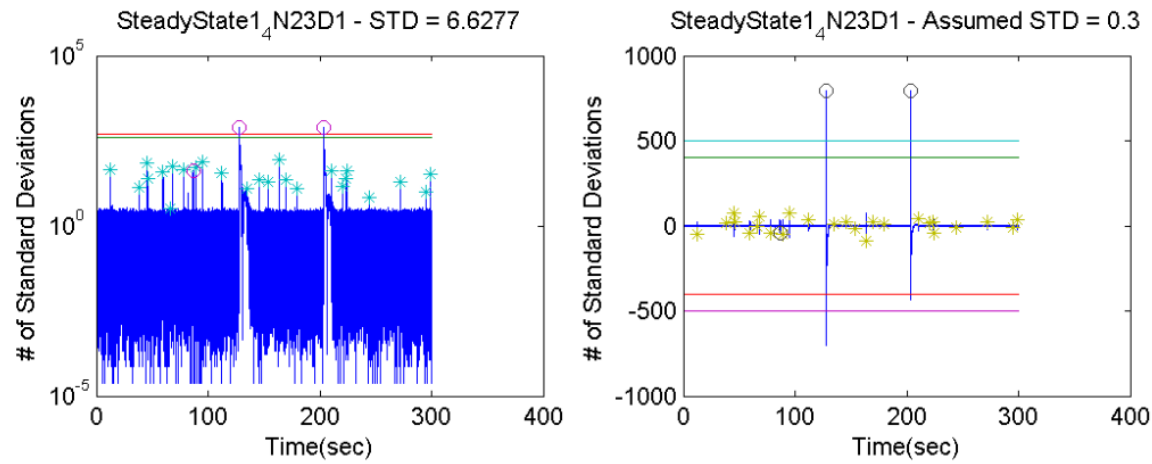

SteadyState1,N23D1 - extinction and migration level pops

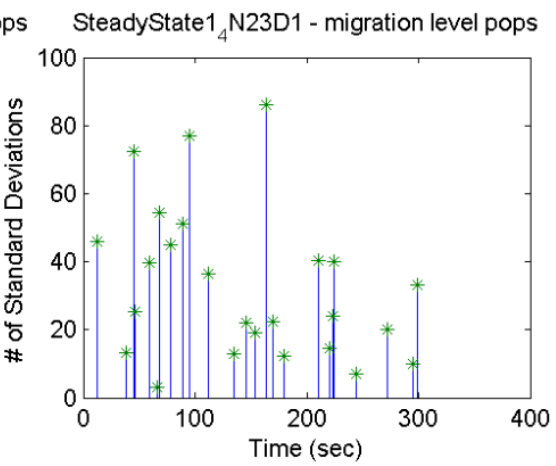

Figure 12. Example Strap Pop Analyses Plots for 23Y from Steady_State1_4

American Institute of Aeronautics and Astronautics 
Figure 13 provides the history of migration-class impulse events (greater than 10 sigma or standard deviation in any one sensor and no values greater than 500 sigma) versus time and pressure. Figure 14 shows the history of the extinction-class events where any one sensor shows an event greater than 500 sigma. In the cases that a data increment was less than five minutes (denoted by the "o" symbol), the number of migration events was scaled up linearly to an approximate number that would have been seen in a five minute data increment. The migration impulse events began to appear when the pressure reached about 1 atmosphere. The rate of events builds up rapidly after that and then appears to plateau. At higher pressures, the rate of migration events falls off as extinction-class events begin to appear in the data.

Figure 15 shows the trace of saturation events where at least one sensor appears to become saturated and unresponsive at some time in the data. A subset of these saturation events are so significant that all sensors on the structure are saturated. Figure 16 illustrates the onset of these global saturation events. One of the most interesting findings of working with the entire 56 segment data set is that these global events are less common and the single channel saturation events are more common than expected from the constant pressure data sets. Figure 17 shows the maximum amplitude event found in any channel of data in each data segment. It is not unexpected that there is a strong correlation between increasing pressure and the max amplitude seen. There is also an important correlation between the ultra-high peaks and the saturation (particularly global saturation) events as seen in Figures 15 and 16.
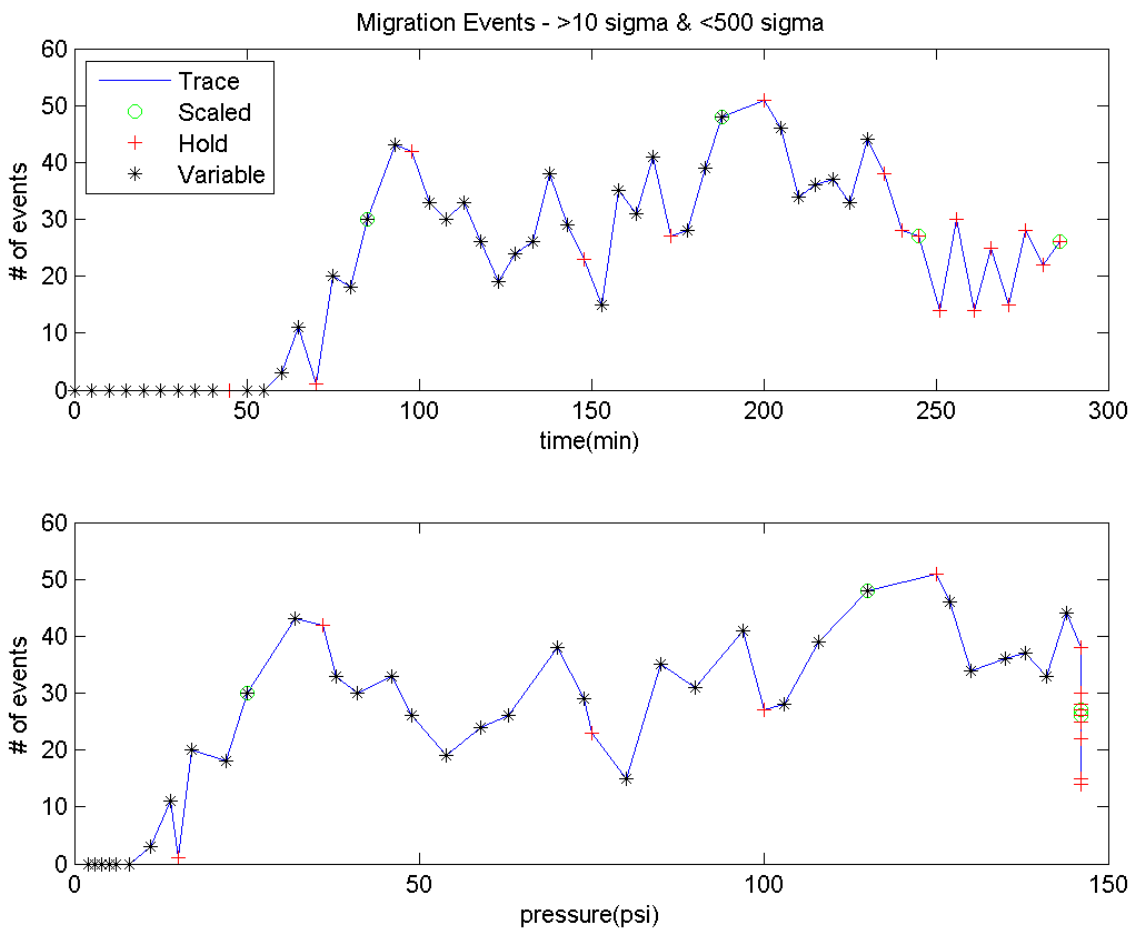

Figure 13. Migration Class Impulse Events vs. Time and Pressure 

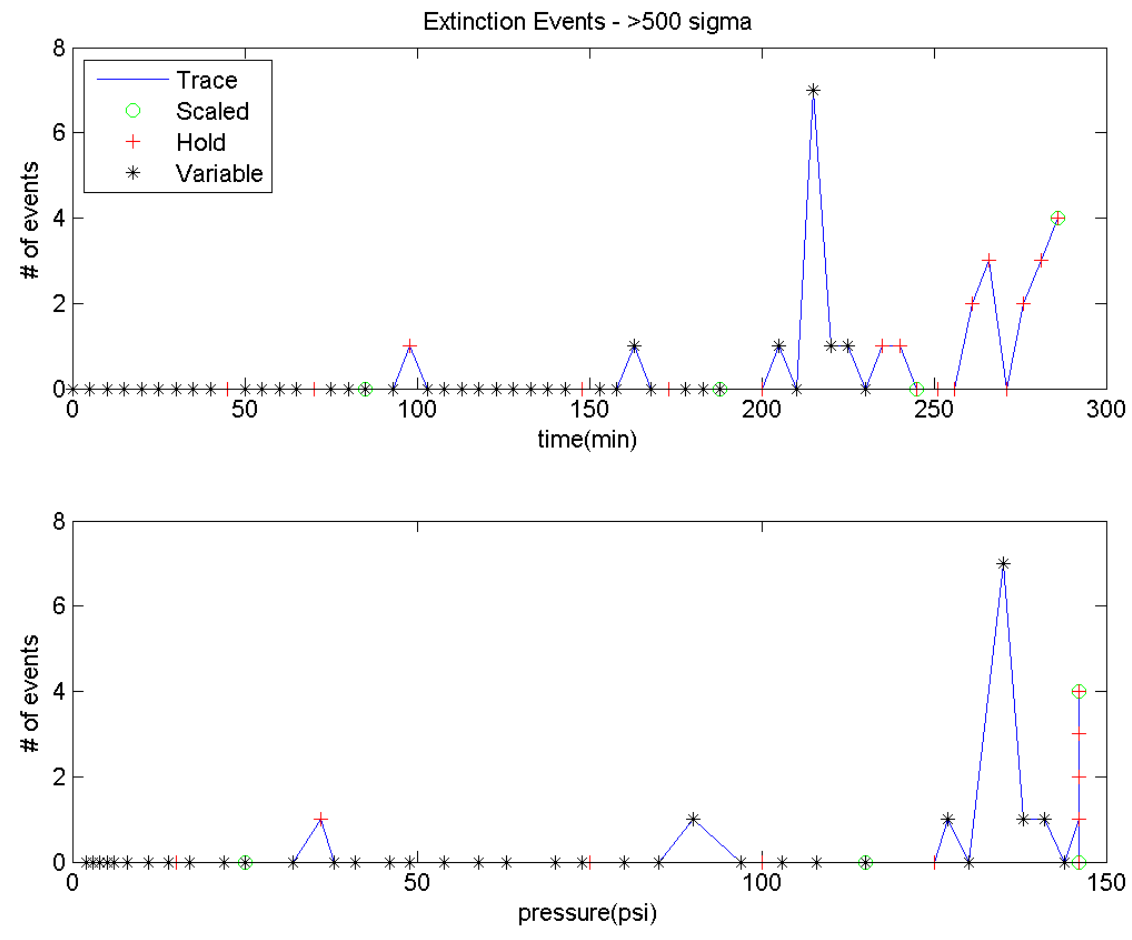

Figure 14. Extinction Class Impulse Events vs. Time and Pressure
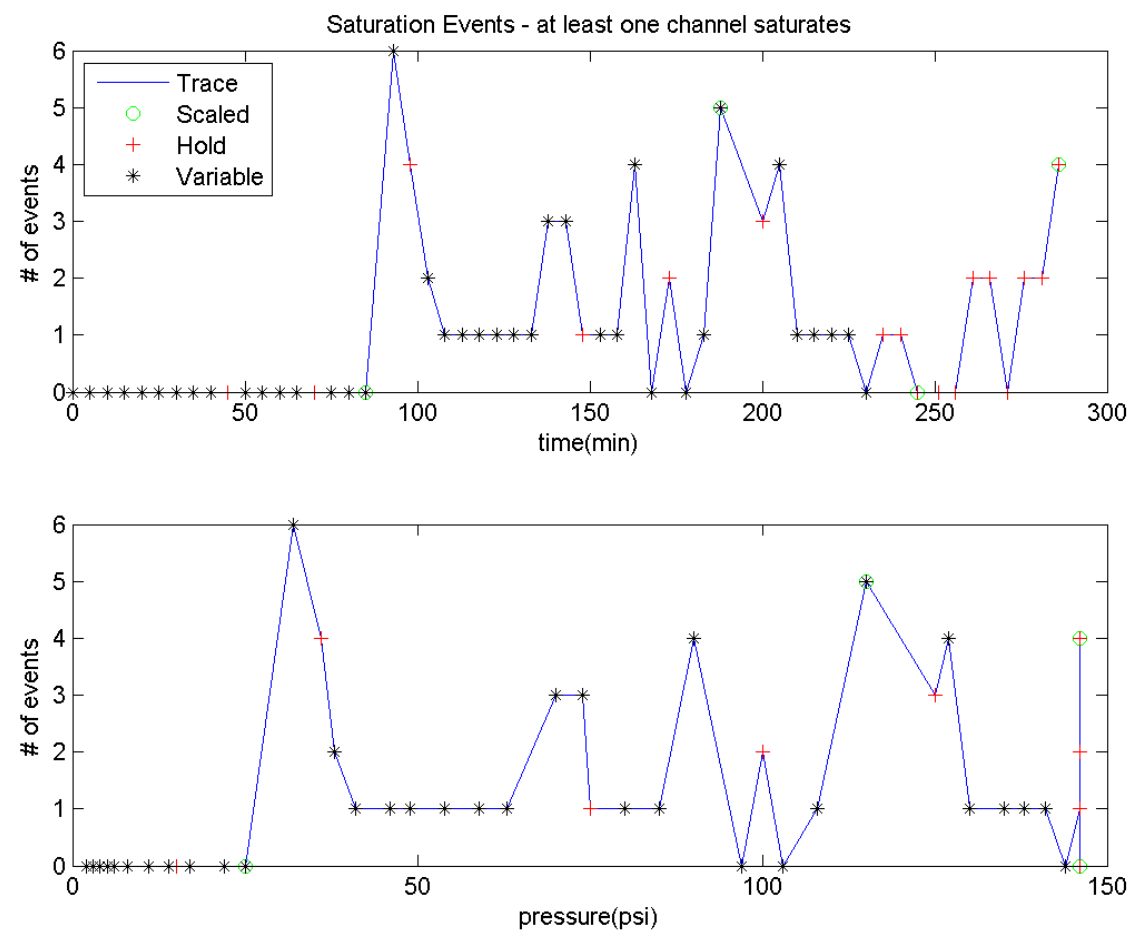

Figure 15. Saturation Class Impulse Events vs. Time and Pressure

American Institute of Aeronautics and Astronautics 

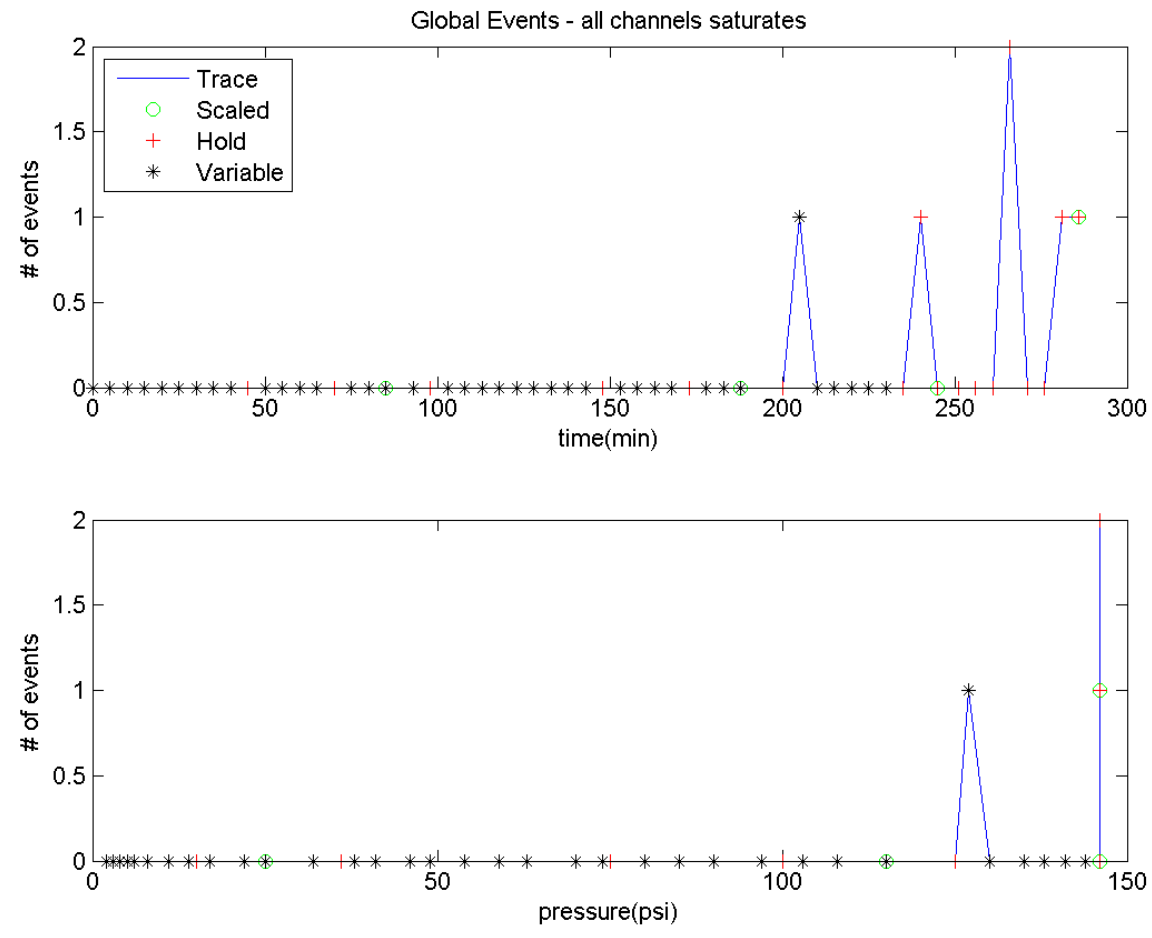

Figure 16. Global Saturation Class Impulse Events vs. Time and Pressure
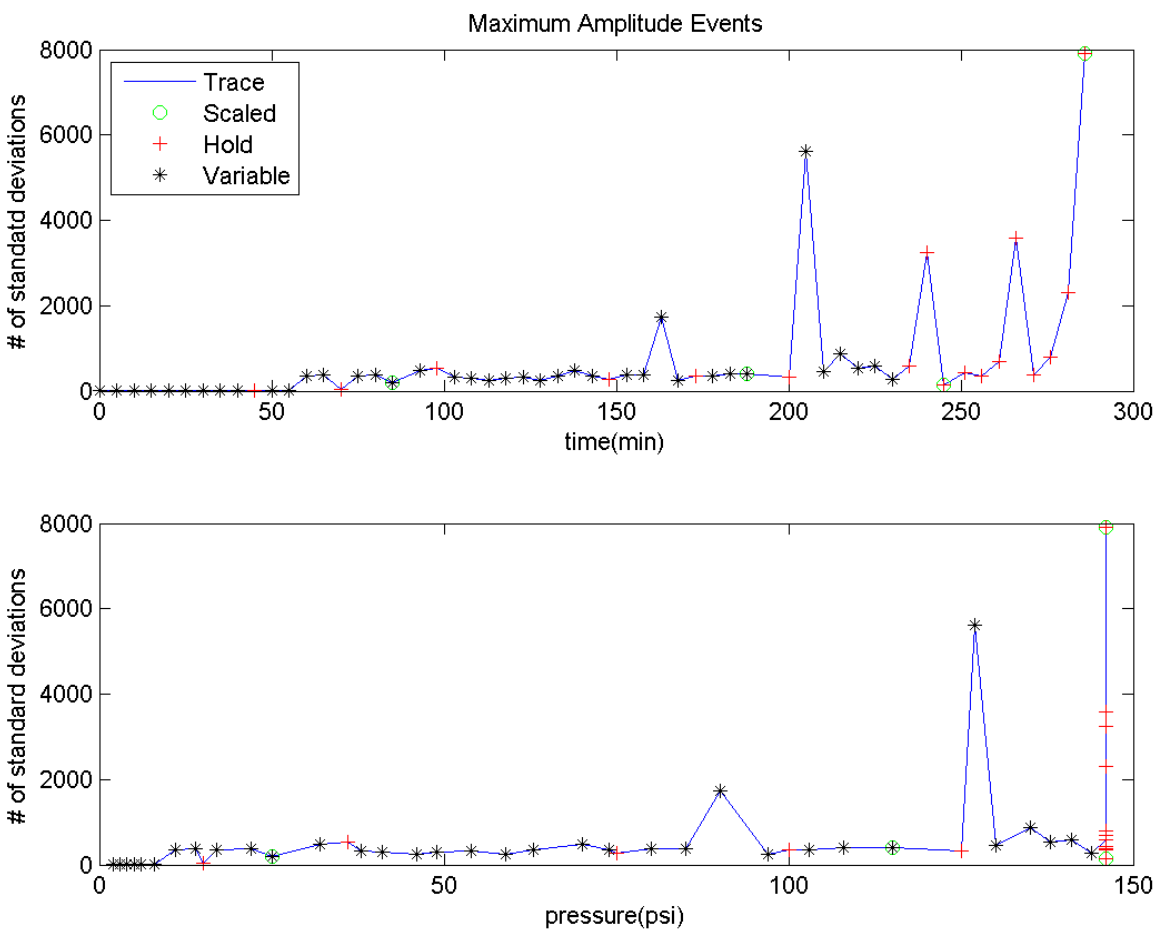

Figure 17. Maximum Normalized Amplitude Impulse Events vs. Time and Pressure 


\section{Potential Explanations}

The high frequency nature of the specific events may be due to sensational effects such as local material failures or just a more mundane explanation that all strap motion under pressure creates a high frequency component that is damped out globally but affects sensors locally. Another explanation is derived from the fact that it was discovered at the beginning of the test that the test frame and/or chamber floor was uneven and a rigid-body rocking mode resulted. Some of the global saturation characteristics might be traced to this mode creating a metal-to-metal impact. An extension of the latter explanation is that many or most of the migration class events could actually have been saturation events if a sensor were closer to the impulse site, but in most cases a sensor was not close enough to the event location to be saturated. These impulse events may also exist on a spectrum, with one extreme being the final failure event. As such an understanding of these events might provide information on the final failure event.

\section{General Modes Assessment}

\section{Transfer Function Approach}

The significant number of impulse events in the data have made the extraction of traditional modes from the data difficult. An approach that has proven to partially successful at modal extraction in the presence of these impulse events has been to estimate Transfer Functions or TFs (may also be called Frequency Response Functions - FRFs) between the sensors mounted on the metallic core and those mounted elsewhere. The transfer functions estimations are developed similar to the following:

$$
\begin{gathered}
T F_{M N}^{T}(\omega)=\left[G_{M M}(\omega)\right]^{-1} *\left[G_{M N}(\omega)\right] ; \\
G_{M N}(\omega)=\left[\begin{array}{ccc}
\Phi_{I_{1}}(\omega) * \Phi_{J_{1}}(\omega) & \cdots & \Phi_{I_{1}}(\omega) * \Phi_{J_{N}}(\omega) \\
\vdots & \ddots & \vdots \\
\Phi_{I_{M}}(\omega) * \Phi_{J_{1}}(\omega) & \cdots & \Phi_{I_{M}}(\omega) * \Phi_{J_{N}}(\omega)
\end{array}\right] ; \\
G_{M M}(\omega)=\left[\begin{array}{ccc}
\Phi_{I_{1}}(\omega) * \Phi_{I_{1}}(\omega) & \cdots & \Phi_{I_{1}}(\omega) * \Phi_{I_{M}}(\omega) \\
\vdots & \ddots & \vdots \\
\Phi_{I_{M}}(\omega) * \Phi_{I_{1}}(\omega) & \cdots & \Phi_{I_{M}}(\omega) * \Phi_{I_{M}}(\omega)
\end{array}\right] ; \text { and } \\
\Phi_{k}(\omega)=\operatorname{FFT}\left\{X_{k}(t)\right\}
\end{gathered}
$$

The following definitions supplement equations (4) - (7):

$\omega$ denotes the frequency domain independent variable;

$I_{m}$ denotes the set of reference indices $(1,2$, and 3 in the work reported herein);

$J_{n}$ denotes the set of response indices (any subset of 1-12 in the work reported herein); and

FFT denotes a Fourier transform.

There are advanced techniques and details such as the alternative uses of cross-and auto-spectra, averaging, overlapping, and windowing that can reduce the effects of noise and other numerical effects that are not discussed her but can be found elsewhere ${ }^{6,7}$.

The Impulse Response Function (IRF) is obtained by taking the inverse Fourier transform of the FRF (or TF).

$$
I R F_{j i}(t)=\operatorname{IFT}\left\{T F_{j i}(\omega)\right\} ; \text { where }
$$

IFT denotes an inverse Fourier transform.

The IRF is a conversion back into the time domain. However, some effects of the forcing function have been reduced, the signal has been windowed and averaged, and the response has been due to any multiple references chosen has been separated. If discrete measurements are assumed then there are advanced algorithms that can be used to develop and use the IRF. It should be noted that the Observer/Kalman filter IDentification or OKID was used for some IRF results presented in this paper ${ }^{8,9}$.

American Institute of Aeronautics and Astronautics 
The top plots of Figures 18 and 19 show these dominant impulse events for two sensors ( $2 \mathrm{X}$ on the metallic core and $41 \mathrm{X}$ on the inflated soft goods of the test article) using 5 minute time records of 1,229,312 samples. The bottom plots show the 614,657 line frequency spectra. These data traces are from the Data Increment SteadyState1_1, which was an early data take at the test pressure of 146 psi. Figure 20 shows the FRF and the IRF due to the $41 X$ response shown in Figure 19 and the 2X reference shown in Figure 18. These calculations were performed to average the data down to 4096 line FRF spectra (2049 are shown in Figure 20). The .5 sec IRF shown in Figure 20 contains 2049 samples. It should also be noted that channels $2 \mathrm{Y}$ and $2 \mathrm{Z}$ were also treated as simultaneous references in the TF estimation and the Figure 20 function is that part of the response that is most correlated to the $2 \mathrm{X}$ reference. It should be noted in Figures 18, 19, and 20 that the dominate frequencies are at $120 \mathrm{~Hz}$ and $1000 \mathrm{~Hz}$, although other distinct resonance peaks are seen in the functions.

A potential next step in the analysis is to assess how these transfer functions change over the course of the test. Figure 21 shows how the same TF shown in Figure 20 varies from the start of the test until failure. The peaks near $1000 \mathrm{~Hz}$ and $120 \mathrm{~Hz}$ appear throughout most of the test. There are a multitude of peaks below $500 \mathrm{~Hz}$ that see to reduce in amplitude after $100 \mathrm{~min}$. There is an increment near 75 seconds that shows some high excitation across the frequency spectra but dominated in the mid-range frequencies of $750 \mathrm{~Hz}$ and below. There are high frequency excitations that appear near the end of the test. Some the low frequency spikes are indicative of that sensor seeing an event that saturates it - although most have been removed from this plot.

\section{Potential Modal Content}

An assessment of the potential modal content in the data was performed using the Impulse Response functions as provided in equation (8). The IRF's were generated using the three of the core measurements as assumed references and all 12 response measurements to generate 36 individual IRFs. These time histories were derived from averaged Frequency Response Functions with a window size of 4096. These 36 IRFs were processed one at a time and the resulting modes were collected to into one superset of modes for each data increment (or sub-increment). The Eigensystem Realization Algorithm with Data Correlations (ERA-DC) as implemented in the SOCIT package was used to generate the estimates ${ }^{9,10}$. The superset of potential modes was filtered using the Consistent Mode Indicator (CMI) using a cut-off of 60 for the data reported below ${ }^{11}$. These results are presented as the first assessment overview to initiate detailed assessments of specific dynamics phenomena.

Figure 22 shows an estimation of the modal content versus time for the range of 2 to $150 \mathrm{~Hz}$. The abscissa of this plot contains the time variable in minutes. The ordinate of the graph contains the estimated frequency of the modes or dynamic phenomena, which were extracted in each data increment. An "*" symbol is provided for each of these estimated frequencies that passed the CMI cut-off criteria. There is a consistent indication of a dynamics phenomenon at $120 \mathrm{~Hz}$. This is most likely a harmonic of 60 cycle noise. However, there does appear to be weak indications of a pressure dependent phenomena in the mid 120's. There are other potentially constant phenomena close to $40 \mathrm{~Hz}$ and above $140 \mathrm{~Hz}$. There are other potentially pressure dependent phenomena that might exist. However the apparent trend that seems to end around $80 \mathrm{~Hz}$ is the strongest potential phenomena. It should be noted that an alternative assessment of modal content to $500 \mathrm{~Hz}$ using only sensors $2 \mathrm{X}, 2 \mathrm{Z}, 22 \mathrm{X}, 22 \mathrm{Z}, 23 \mathrm{X}$, and $23 \mathrm{Z}$ suggested the following frequencies were expected: $45 \mathrm{~Hz}, 60 \mathrm{~Hz}, 80 \mathrm{~Hz}, 115 \mathrm{~Hz}, 120 \mathrm{~Hz}$, and $140 \mathrm{~Hz}$.

Figure 23 shows the same type of information for the range of $150 \mathrm{~Hz}$ to $300 \mathrm{~Hz}$. While there are potential indications of phenomena at several frequencies, the only one of note is close to $240 \mathrm{~Hz}$. However, this is suspicious as it is twice the $120 \mathrm{~Hz}$ phenomena and also a harmonic of 60 cycles. Such harmonics are common noise phenomena in electronic measurements. The alternative assessment noted above suggested the following frequencies were near candidate modes: $160 \mathrm{~Hz}, 175 \mathrm{~Hz}, 180 \mathrm{~Hz}, 195 \mathrm{~Hz}, 200 \mathrm{~Hz}, 220 \mathrm{~Hz}, 235 \mathrm{~Hz}, 240 \mathrm{~Hz}, 255 \mathrm{~Hz}, 260 \mathrm{~Hz}, 280 \mathrm{~Hz}$, and $298 \mathrm{~Hz}$.

Figure 24 carries the discussion to the $300 \mathrm{~Hz}$ to $600 \mathrm{~Hz}$ range. A potential phenomena near $360 \mathrm{~Hz}$ is also suggestive of higher harmonics of 60 cycle noise. However, at the low pressures there is a potential phenomenon close to $540 \mathrm{~Hz}$ that is fairly consistent. It should be noted that in all of these frequency scatter plots, there are a large number of additional frequencies estimated when the first strong impulse excitation events occur at around 93 minutes into the test. This frequency range is specifically devoid of strong trends. This violates the pre-test expectations that significant ovalling modes should be present in the pressurized softwoods of the test article. However, this expectation has been modified by the hypothesis that the impulse events do not allow this class of modes to be consistently excited. Although the alternative assessment had found frequency content at: $335 \mathrm{~Hz}, 360 \mathrm{~Hz}, 375 \mathrm{~Hz}, 385 \mathrm{~Hz}, 400 \mathrm{~Hz}, 430 \mathrm{~Hz}$, $445 \mathrm{~Hz}, 480 \mathrm{~Hz}$, and $495 \mathrm{~Hz}$.

Figure 25 finishes the data provided in this scatterplot form with the $600 \mathrm{~Hz}$ to $1100 \mathrm{~Hz}$ range of data. This plot shows a pair of fairly consistently excited phenomena near $1000 \mathrm{~Hz}$ and $1025 \mathrm{~Hz}$. These phenomena appear to be time dependent not pressure dependent. The $1000 \mathrm{~Hz}$ phenomena was studied in more detail and will be discussed next.

American Institute of Aeronautics and Astronautics 

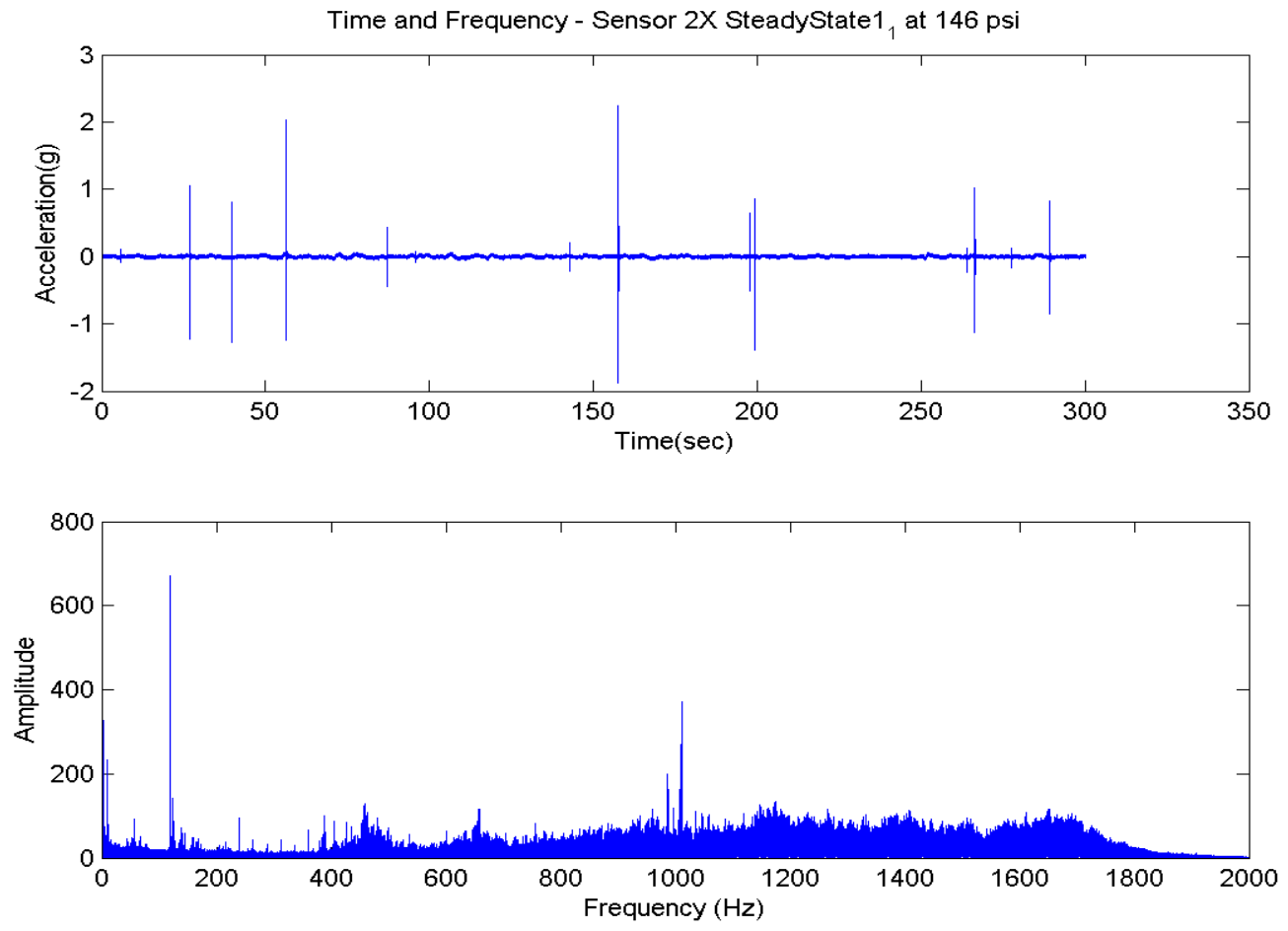

Figure 18. Time History and Frequency Spectra of Core Sensor 2X - SteadyState1_1 Increment
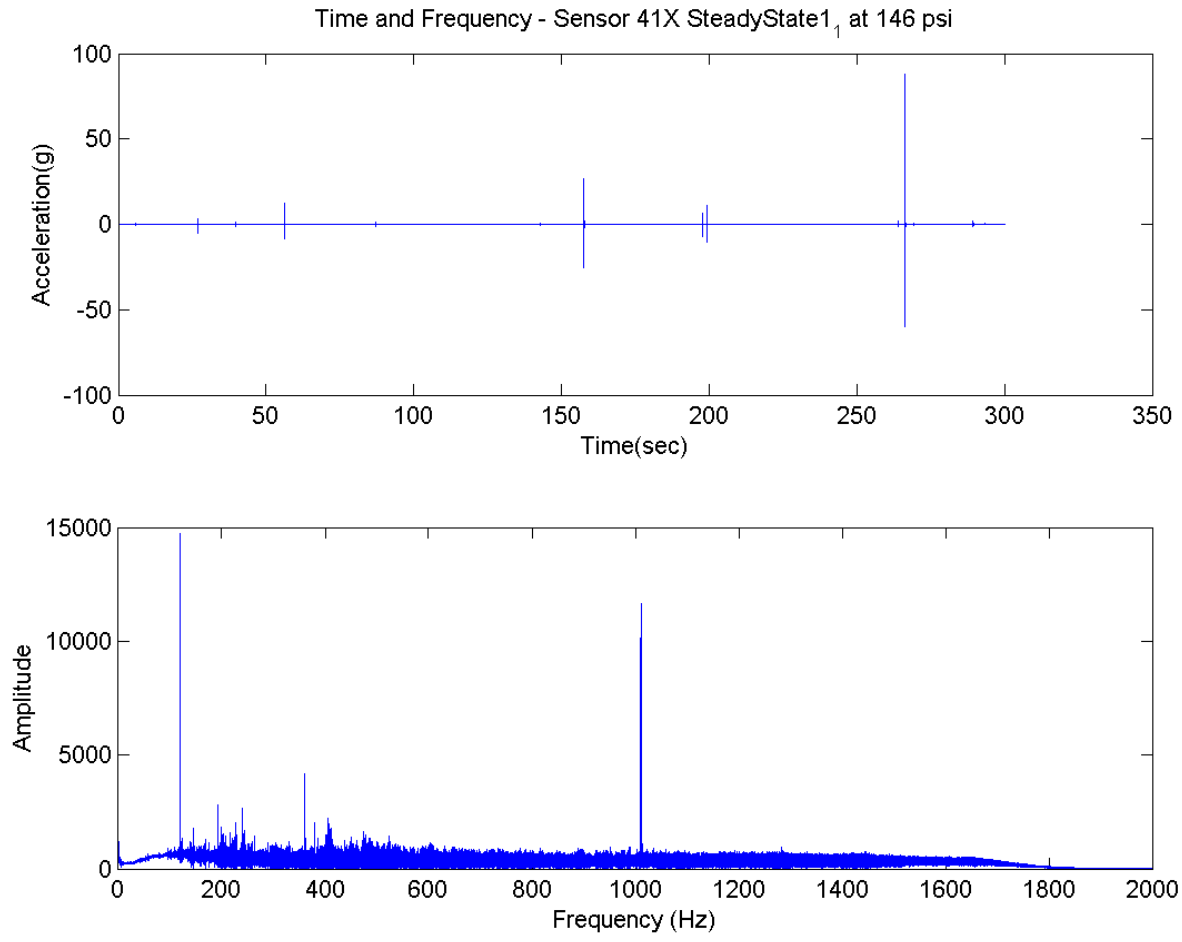

Figure 19. Time History and Frequency Spectra of Soft Goods Sensor 41X - SteadyState1_1 Increment 

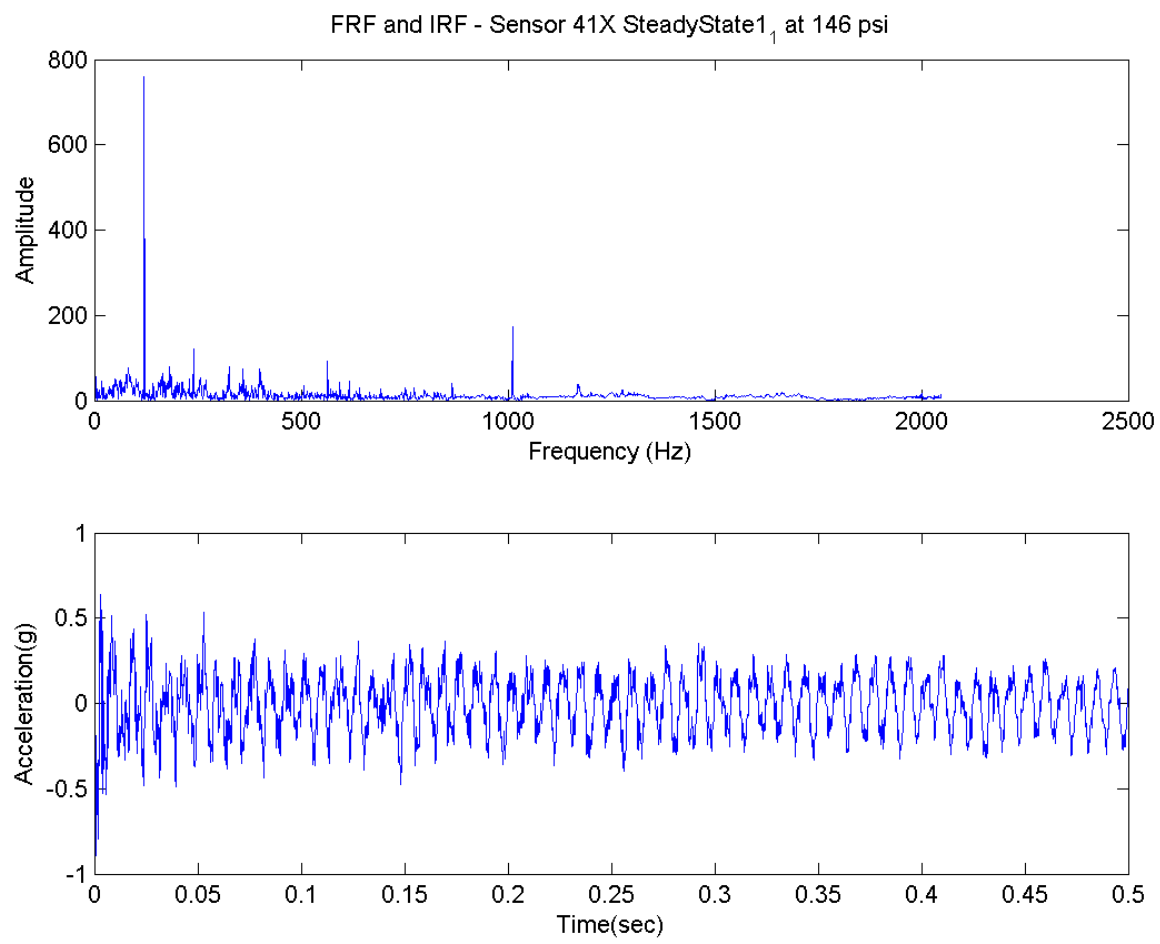

Figure 20. $2 X$ to 41X Transfer Function and Impulse Response Function - SteadyState1_1 Increment

Transfer Function vs. Time - Reference 2X+ - Response 41X-

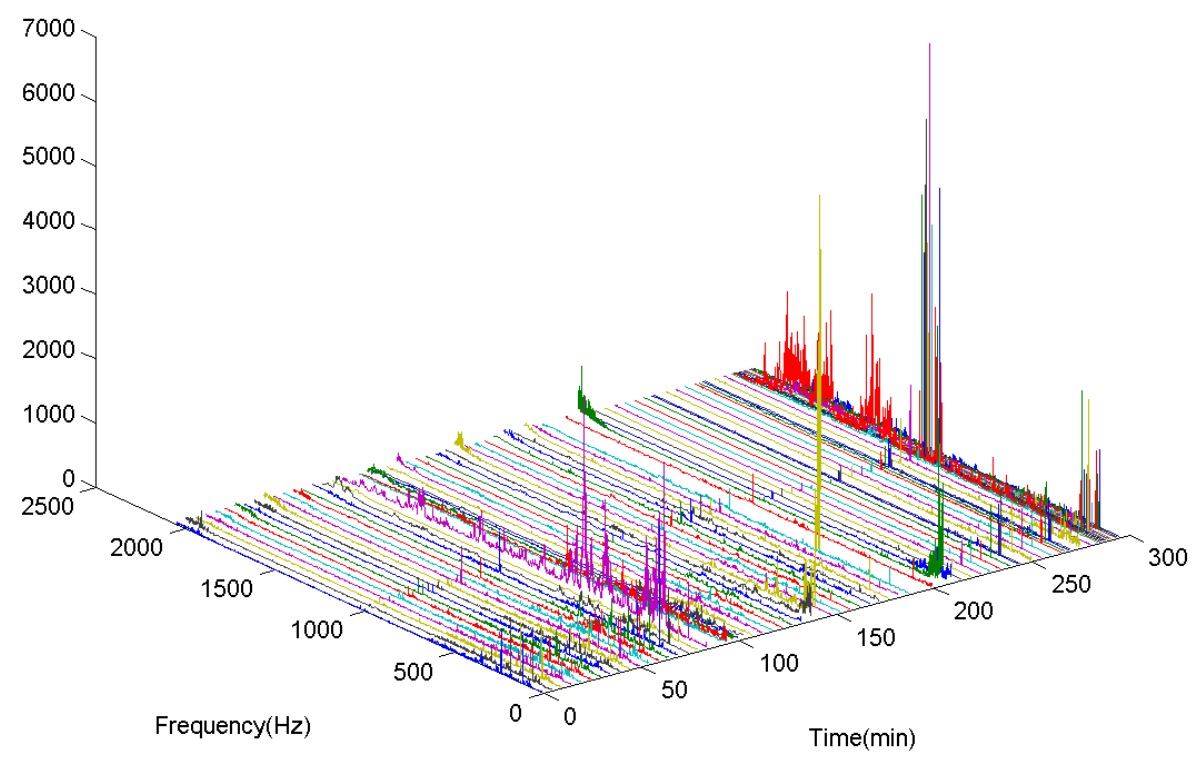

Figure 21. $2 \mathrm{X}$ to $41 \mathrm{X}$ Transfer Function versus Time 


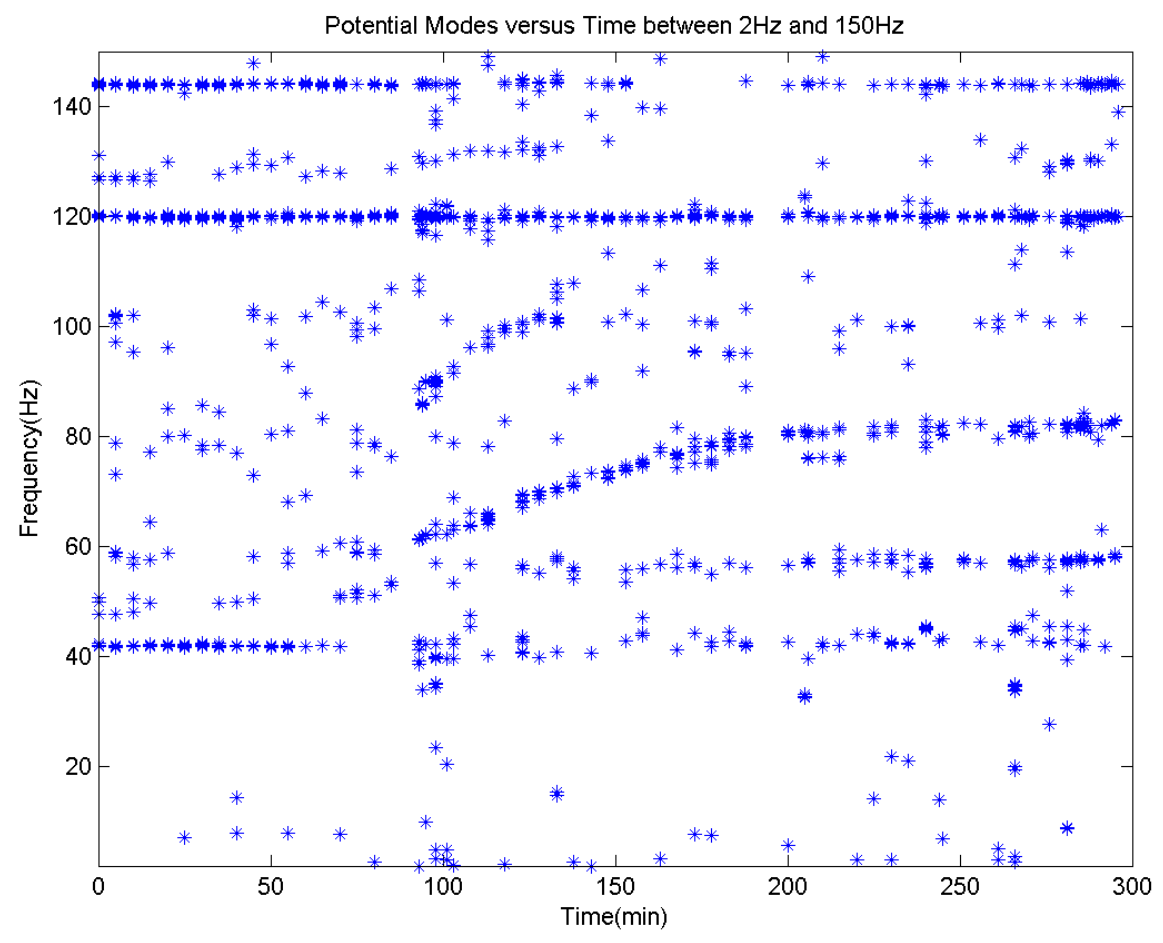

Figure 22. Potential Modal Content versus Time from $2 \mathrm{~Hz}$ to $150 \mathrm{~Hz}$

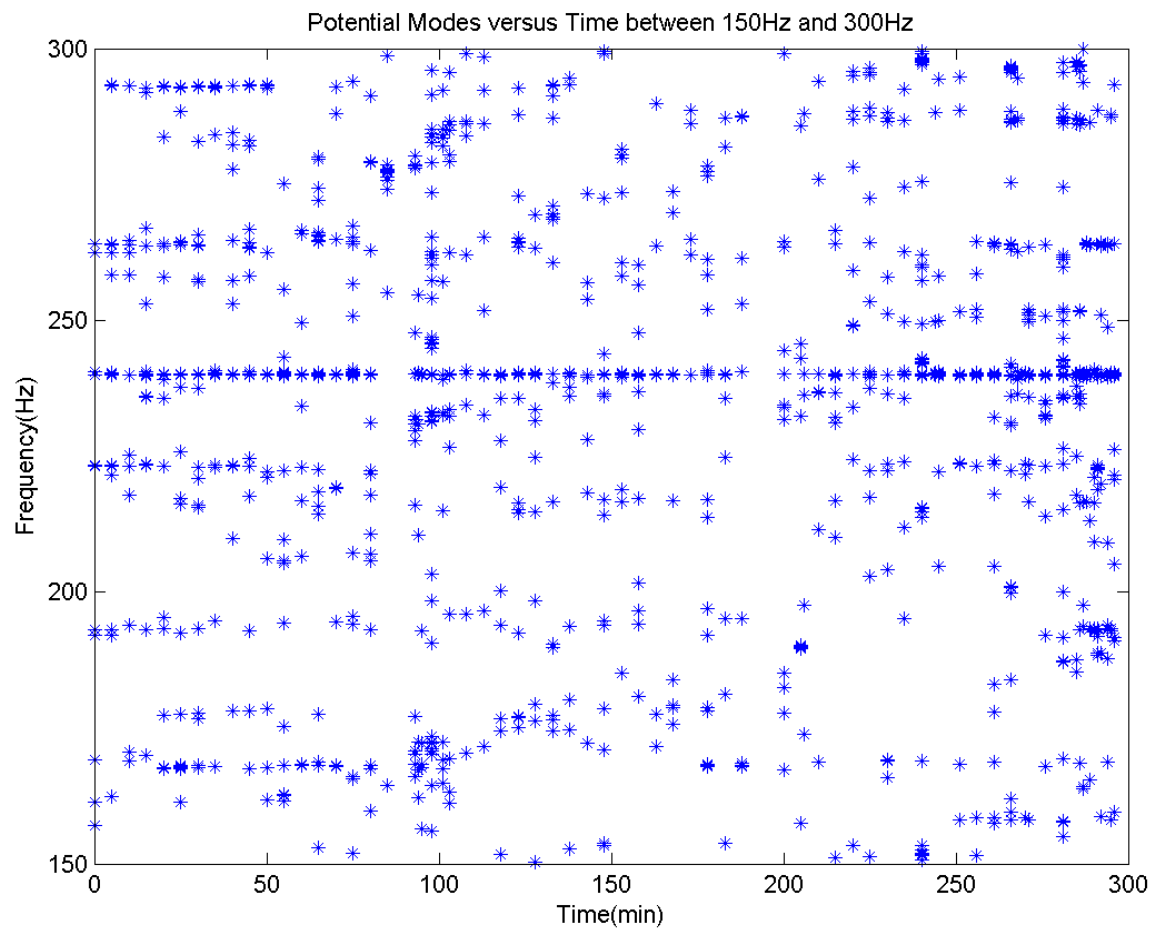

Figure 23. Potential Modal Content versus Time from $150 \mathrm{~Hz}$ to $300 \mathrm{~Hz}$

American Institute of Aeronautics and Astronautics 


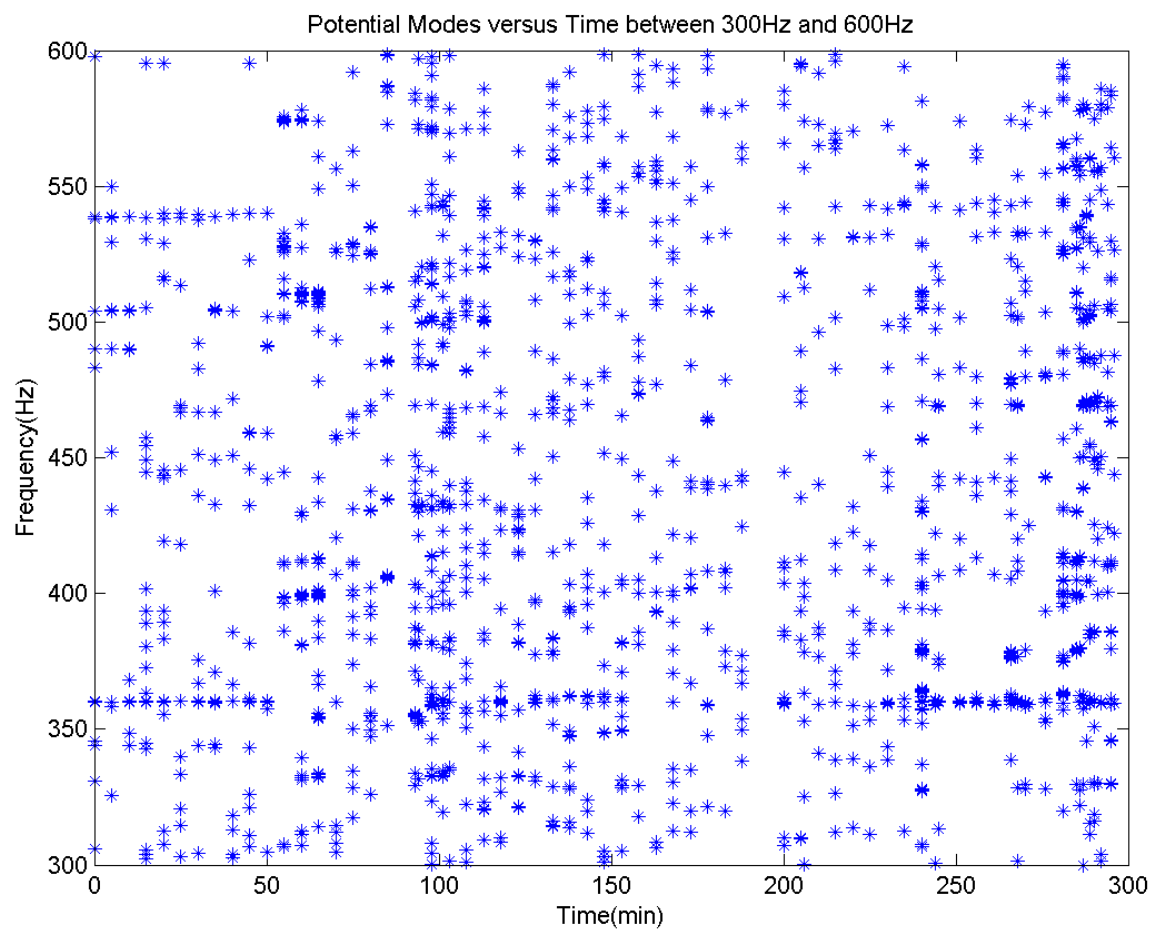

Figure 24. Potential Modal Content versus Time from $300 \mathrm{~Hz}$ to $600 \mathrm{~Hz}$

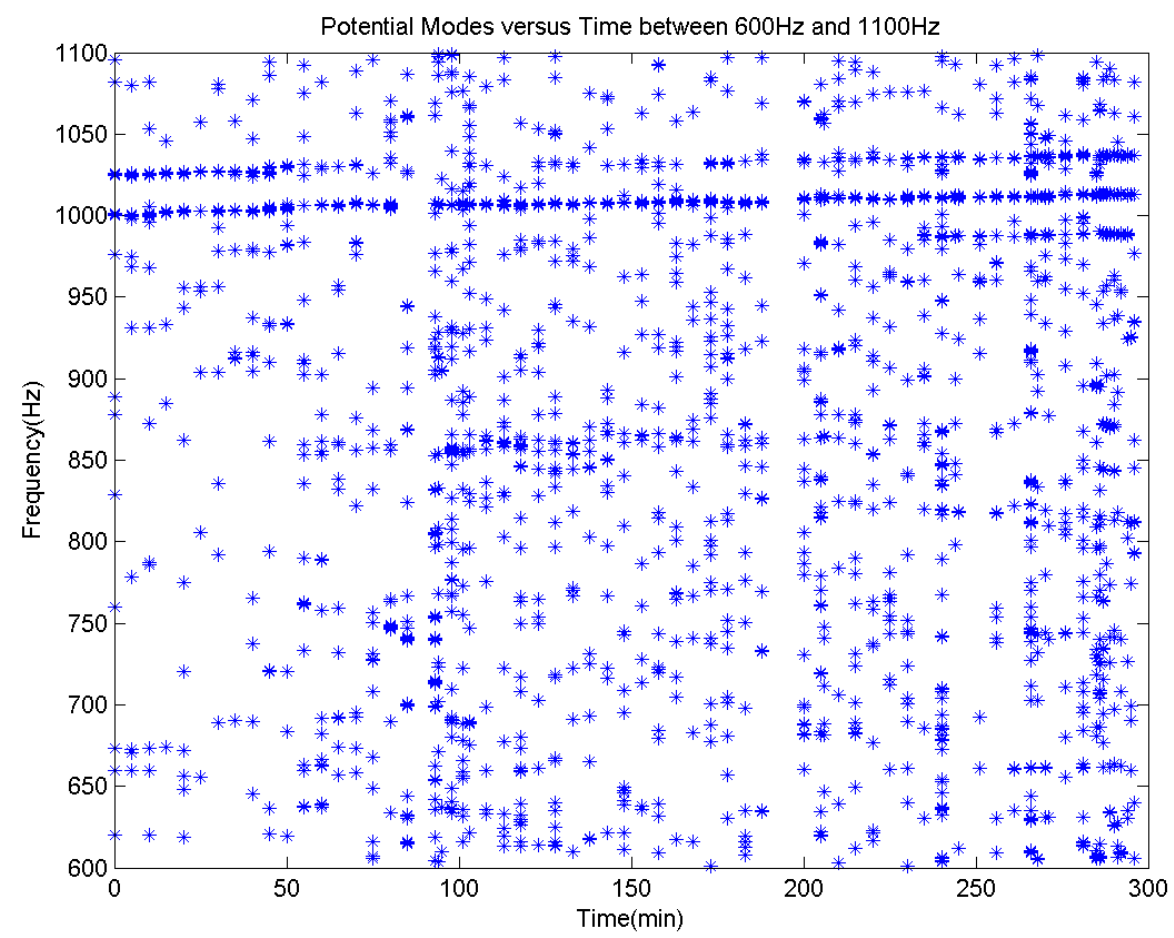

Figure 25. Potential Modal Content versus Time from $600 \mathrm{~Hz}$ to $1100 \mathrm{~Hz}$

American Institute of Aeronautics and Astronautics 


\section{$1000 \mathrm{~Hz}$ Assessment}

\section{Assessment of Frequency Trends}

The $1000 \mathrm{~Hz}$ phenomena is a narrow band vibration that appears strongly in the final data sets of the test and can be found throughout the data set, as shown in Figures 21 and 25. The transfer function approach discussed above is used to extract the information on this phenomena. The MIDOS software ${ }^{1}$ was used to extract the frequency and damping of the phenomena from all of the variable and constant pressure data sets. Two extractions were performed, each of which used one reference and response function pair per data increment. In all cases, one of the three sensors on the metallic core were used as the reference sensor. In the first case (labeled as Max Sensors), the reference/response pair was selected for each data increment with the most prominent peak near $1000 \mathrm{~Hz}$. This assured the best potential frequency extraction from each data increment. The Max Sensors extraction also demonstrated the frequency consistency in all 12 measurements as all sensors used in at least one and usually the reference/response pair selected varied from one data increment to the next. In second case (labeled as Max Consistency), the ref/res pair was always set at a reference of $2 \mathrm{x}$ and a response of $41 \mathrm{x}$. This assured maximum consistency from one data increment to the next. Note that Figure 21 is a visualization of the data set used for the Max Consistency processing. Reporting on the similarity and differences of these two analyses provides a check on the data analysis process and the consistency of the results. Additionally the Max Consistency set split the final 70 second data increment with the run-up to terminal failure into ten sub-increments.

The sensor saturation effects can have a significant impact on the data processing, even when using the transfer function approach. Figure 12 above does show some of the effects of sensor saturation. Figure 26 shows these effects in greater detail with an expanded time plot and a frequency domain version of the data. Note that in the frequency domain, saturation will create significant low frequency content.

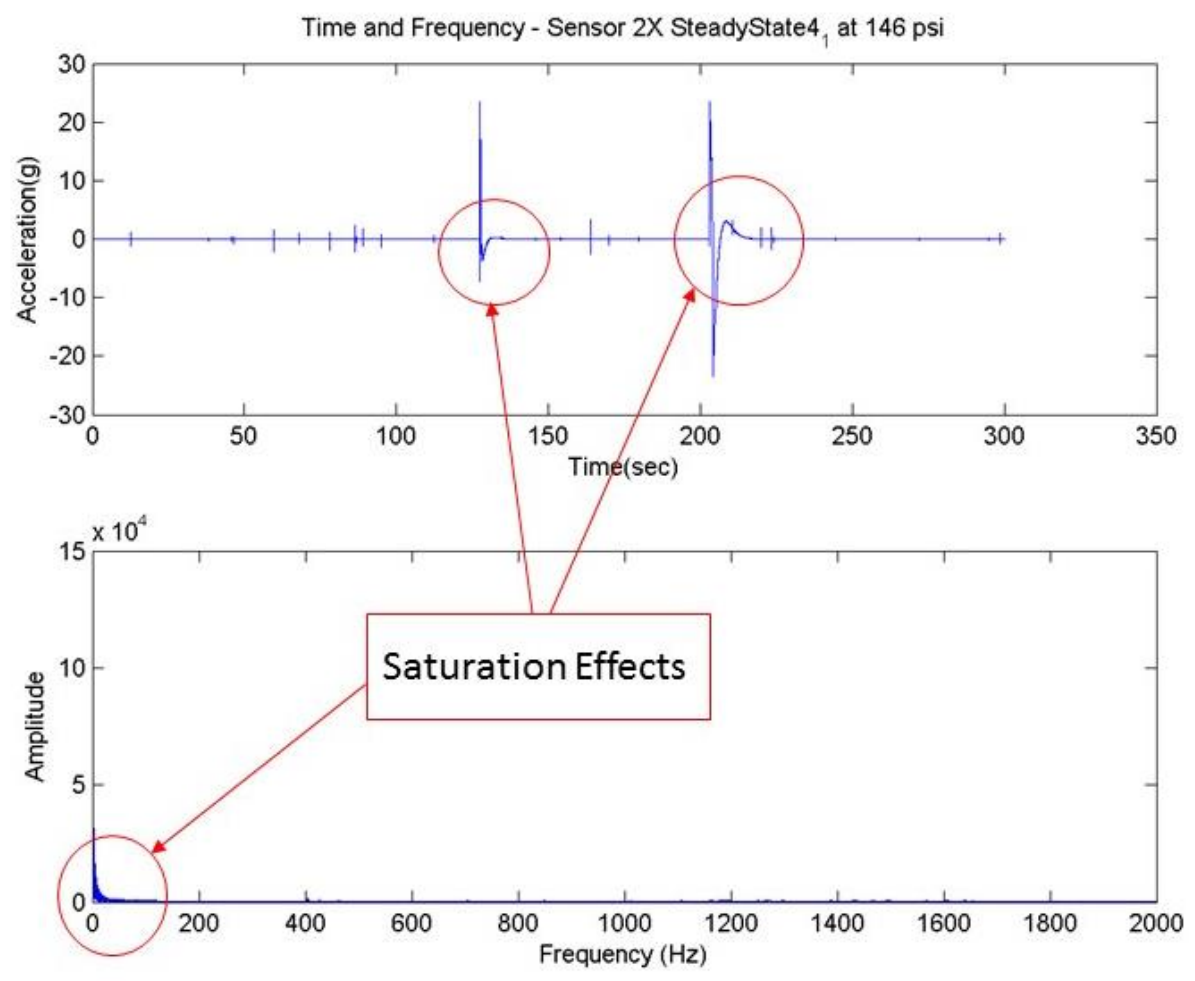

Figure 26. Time History and Frequency Spectra of Saturated Sensor 2X - SteadyState1_4 Increment

Figures 27 and 28 show the frequency trends of the $1000 \mathrm{~Hz}$ phenomena with respect to time and pressure. The Max Sensors data are the black symbols, while the Max Consistency frequency estimates are given as the red symbols. The processing to extract the $1000 \mathrm{~Hz}$ phenomena data was performed on the complete data set that may have contained saturated sensors. When a data increment contained global saturation effects, the data was also processed for sub-increments of the data that did not include the global saturation effects. In Figures 27 and 28 , the

American Institute of Aeronautics and Astronautics 
"o" symbols denote a frequency estimate from complete data increments that contained at least one global saturation event. The remainder of the data are from increments and sub-increments that have had the global saturation events removed. It should be noted that any single sensor saturation event effects were not removed from the processing.

There is a definitive trend of increasing frequency for this phenomena throughout the test. Hence, further study of this phenomena may uncover a metric for the structural health of the test article. The initial rise to $1006 \mathrm{~Hz}$ appears to be more strongly correlated to pressure, while time appears to be the dominate parameter for the remainder of the test. There does not appear to be a significant difference in frequency trend between the Max Sensors or the Max Consistency processing. Nor do the global saturation events appear to affect the data processing significantly. The data averaging inherent in the use of IRFs may be a factor in reducing the effects of the sensor saturation.

\section{Assessment of Amplitude Trends}

The Max Consistency set frequency and damping values were used to perform a least squares fit of a damped cosine, a damped sine function, and a constant offset to a full set of Impulse Response Functions (IRFs). The amplitude estimation process for a single increment due to response $j$ and input $i$ can be restated mathematically as follows:

$$
\begin{gathered}
A_{k j i}=\sqrt{S_{j i}^{2}+C_{j i}^{2}} ; \\
\left\{\begin{array}{c}
S_{k j i} \\
C_{k j i} \\
O_{k j i}
\end{array}\right\}=\left(W^{T} W\right)^{-1} * W^{T} * I R F_{k j i} ; \text { and } \\
W=\left[\begin{array}{ccc}
e^{-\xi_{k} \omega_{k} t_{1}} \sin \left(\omega_{f} t_{1}\right) & e^{-\xi_{k} \omega_{k} t_{1}} \cos \left(\omega_{f} t_{1}\right) & 1 \\
\vdots & \vdots & \vdots \\
e^{-\xi_{k} \omega_{k} t_{P}} \sin \left(\omega_{f} t_{Q}\right) & e^{-\xi_{k} \omega_{k} t_{P}} \cos \left(\omega_{f} t_{Q}\right) & 1
\end{array}\right] .
\end{gathered}
$$

Equations (9) - (11) utilize the following definitions:

$\omega_{k}$ is the frequency of the $1000 \mathrm{~Hz}$ phenomena extracted for the kth increment;

$\xi_{k}$ is the damping ration of the $1000 \mathrm{~Hz}$ phenomena extracted for the kth increment; and

$t_{Q}$ is the final time used for the estimation.

To drive these estimations, 36 IRFs were generated by assuming all three core measurement sensors as the references and all 12 sensors (which also included the core measurements) as responses. This IRF set also included averaging to estimate 4096 line transfer functions producing 2049 IFR time points. The results were amplitude estimates of the $1000 \mathrm{~Hz}$ phenomena over time. Figure 29 provides the amplitude results for response sensor 41X. Plots for the sensor results with respect to each of the three assumed references. The increments without global saturation, the increments with global saturation, and the sub-increments to remove global saturation are denoted. Trace lines for visibility are provided. Also there are short lines provided, which denote three times when no data acquisition was occurring to so a software restart. The amplitude does rise drastically near failure. It should be noted that there are 10 sub-increments of approximately 6.7 seconds each to assess the run-up to failure in more detail. In order to assess the other three sensors on the face of the test article soft goods consistent with 41X, Figures $30(31 \mathrm{X}), 31(21 \mathrm{X})$, and $32(51 \mathrm{X})$ are provided. It should be also noted that the final failure initialization has not been identified, however the indications are that it occurred near the region covered by these four sensors ${ }^{2}$.

American Institute of Aeronautics and Astronautics 


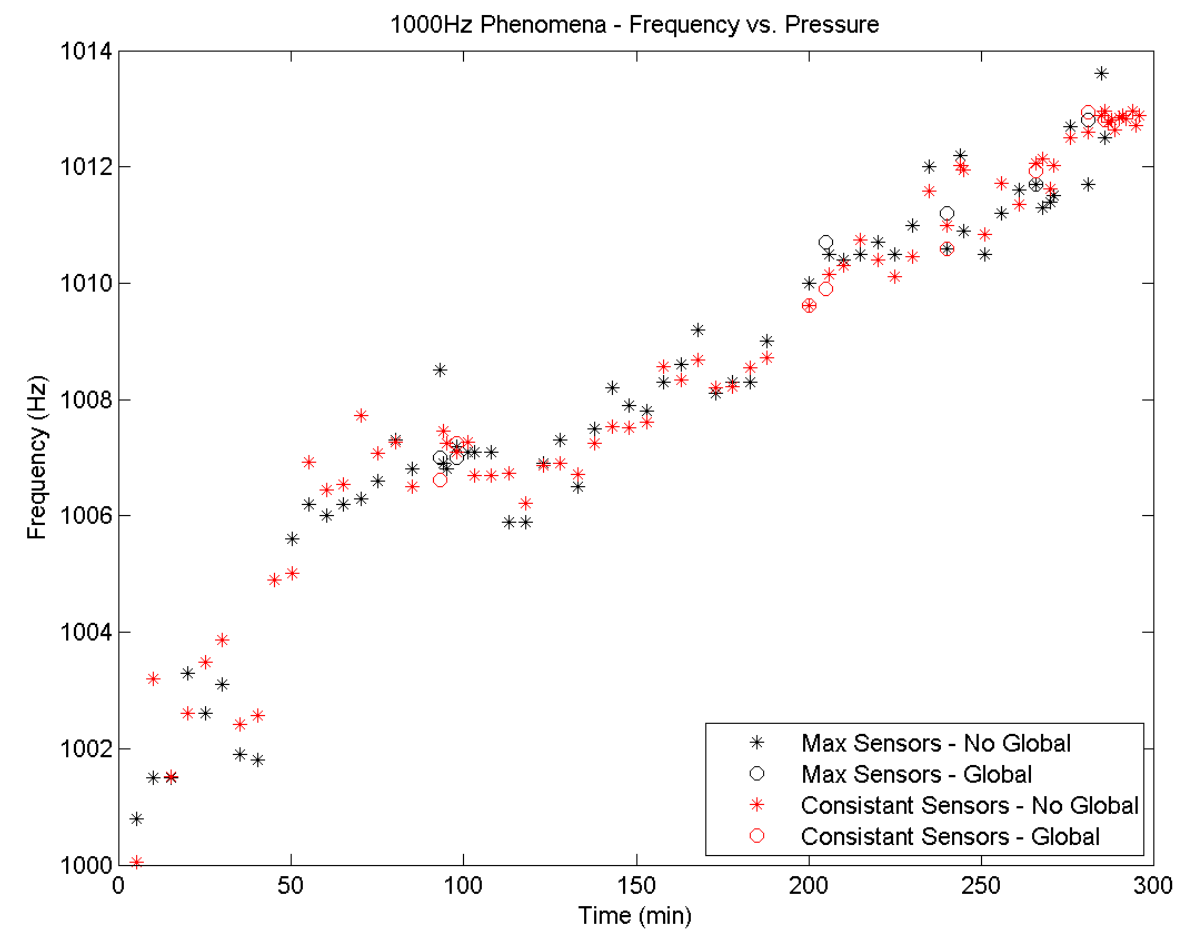

Figure 27. Frequency Trends Versus Time for the $1000 \mathrm{~Hz}$ Phenomena

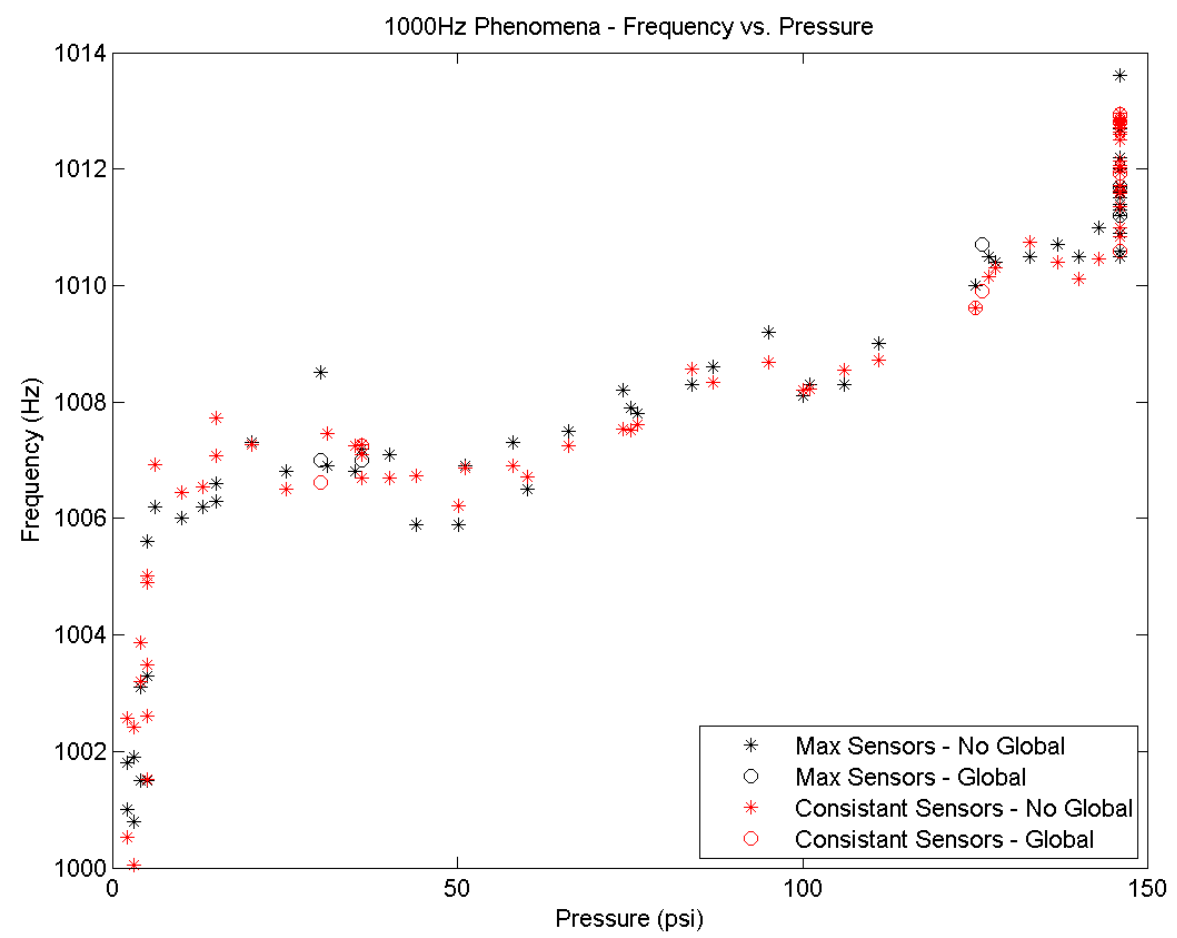

Figure 28. Frequency Trends Versus Pressure for the $1000 \mathrm{~Hz}$ Phenomena

American Institute of Aeronautics and Astronautics 

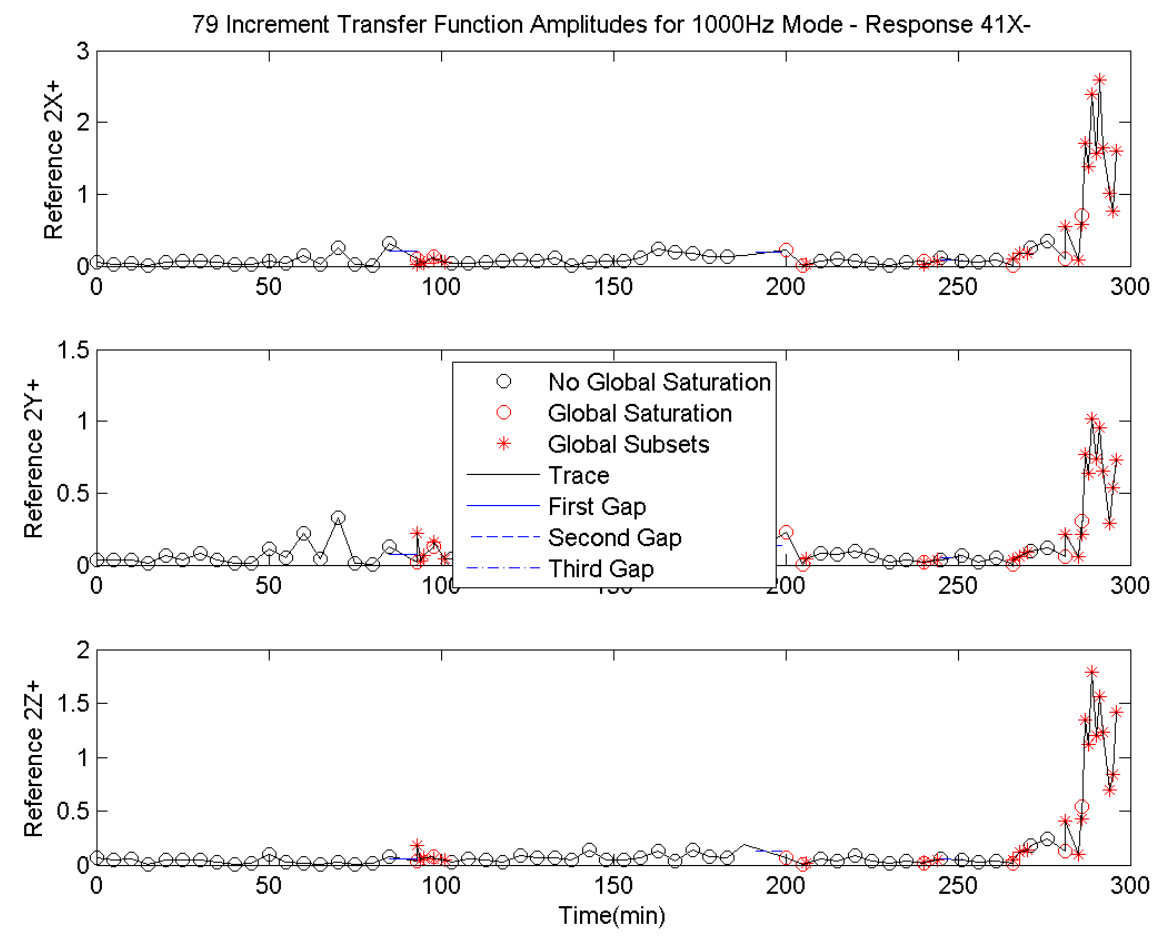

Figure 29. Max Consistency Amplitude Trends in Sensor 41X Versus Time for the $1000 \mathrm{~Hz}$ Phenomena
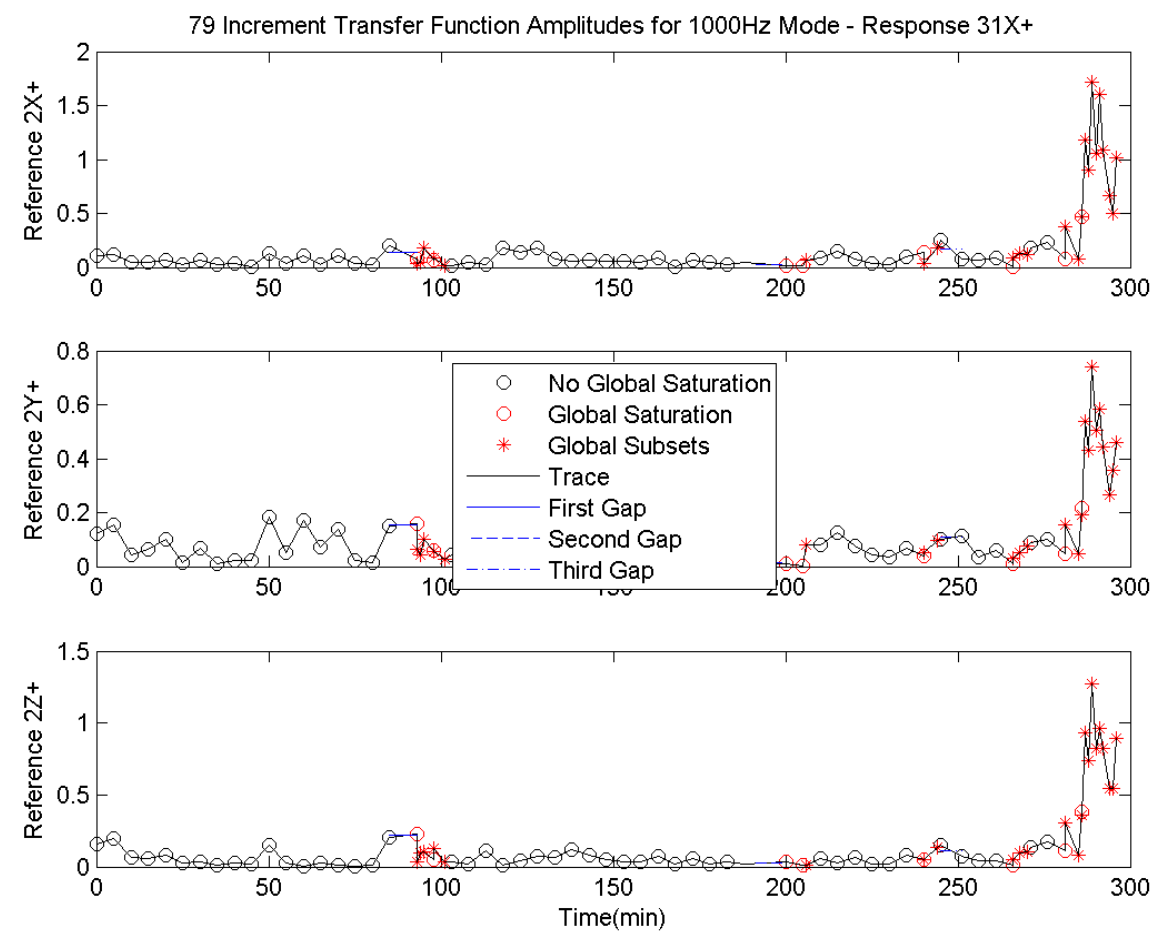

Figure 30. Max Consistency Amplitude Trends in Sensor 31X Versus Time for the $1000 \mathrm{~Hz}$ Phenomena

American Institute of Aeronautics and Astronautics 

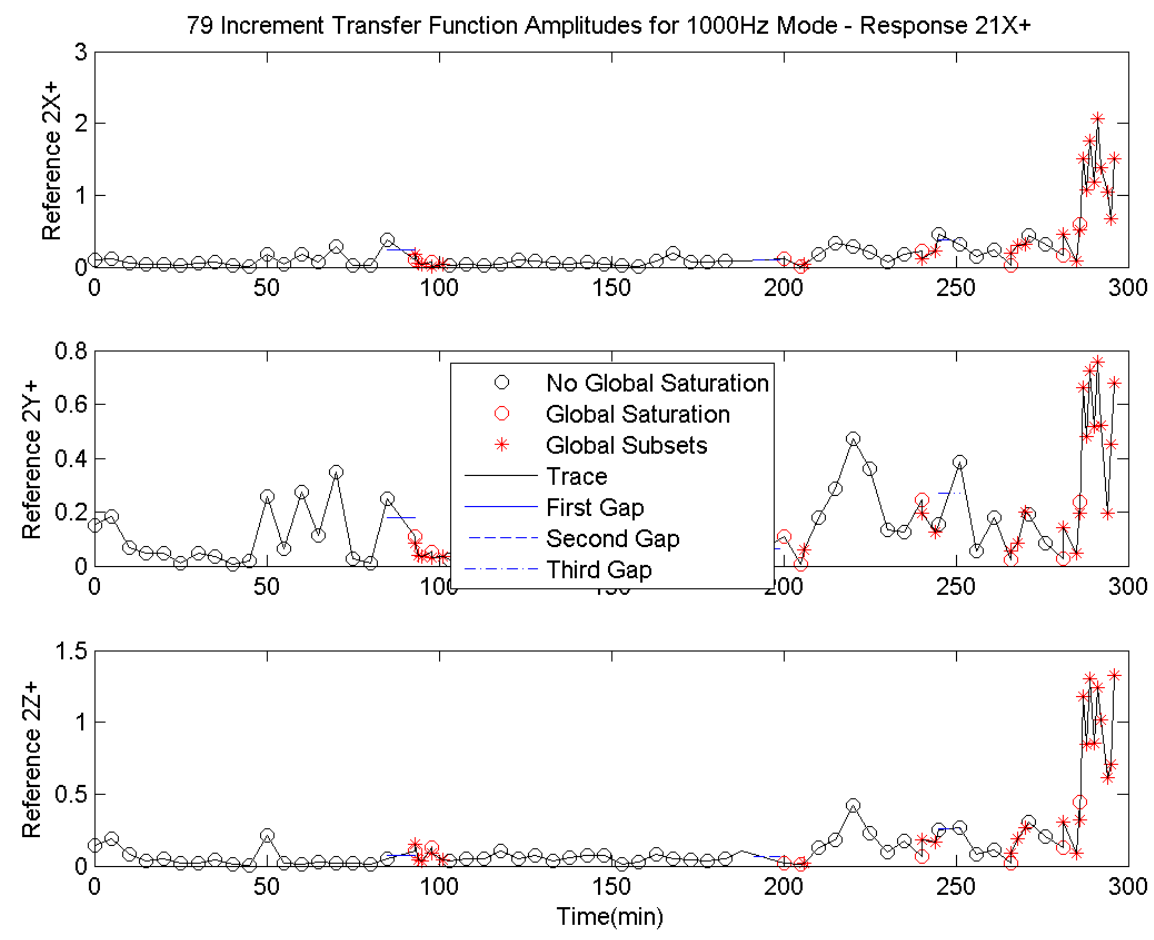

Figure 31. Max Consistency Amplitude Trends in Sensor 21X Versus Time for the $1000 \mathrm{~Hz}$ Phenomena
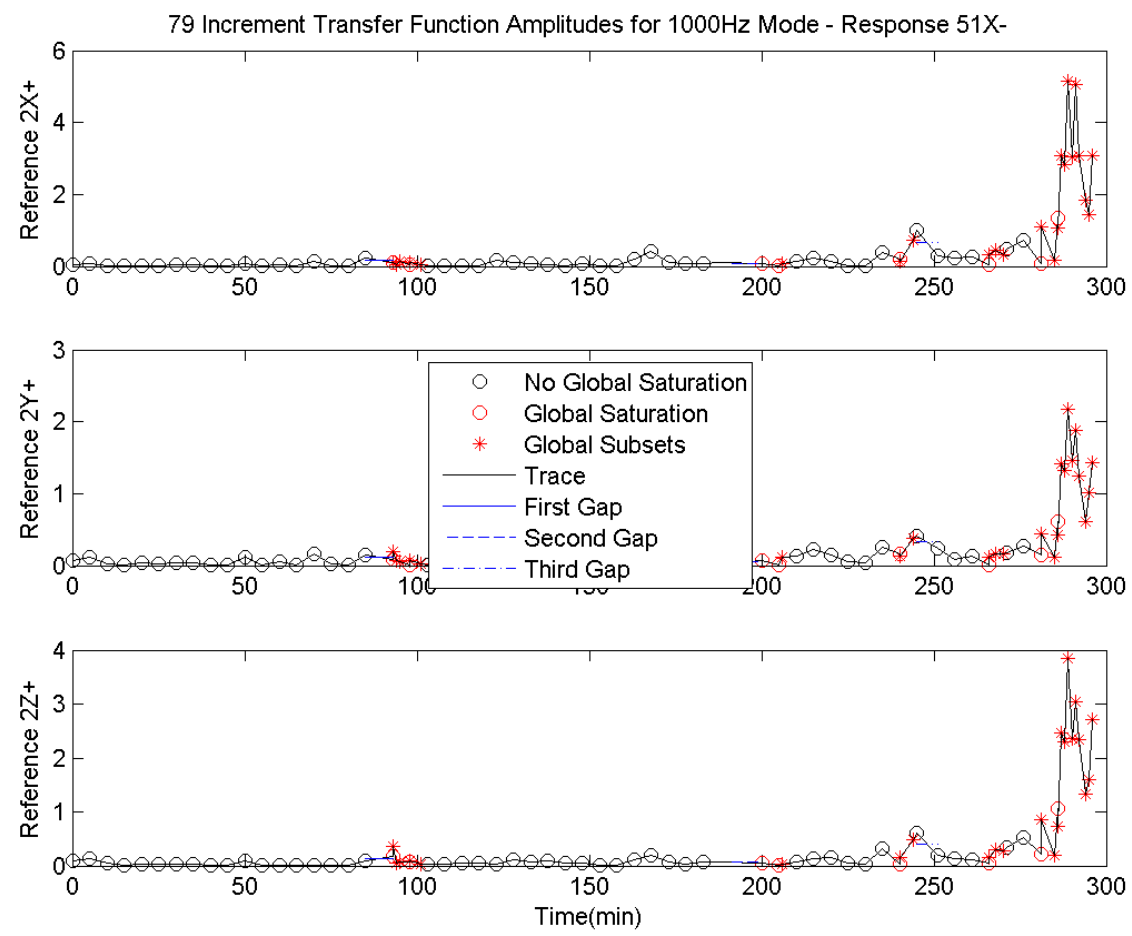

Figure 32. Max Consistency Amplitude Trends in Sensor 51X Versus Time for the $1000 \mathrm{~Hz}$ Phenomena

American Institute of Aeronautics and Astronautics 


\section{Future Work}

The work reported herein is supportive of an analysis plan that was generated for the assessment of the accelerometer data from this inflatable creep test. Round 1 of the suggested work focused on assessments of the data without significant analytical or advanced algorithm support. Round 2 adds analytical modelling to the effort as well as advanced algorithms such as Ritz vector estimation ${ }^{4}$ and force estimation ${ }^{5}$. Round 3 couples these different techniques together and also applies advanced damage identification algrothms ${ }^{12}$. This work is contained in Round 1 of this plan.

A major item of unfinished work in Round 1 is the analysis of the modal test data that preceded this work. This modal test data would be an anchor for the modal assessments in the test configuration as well as for the analytical models of the test article.

Of course, there is much more data analysis that should be performed on the data as-is. Additional efforts have been defined to add more of the low amplitude modal content to the frequency scatter plots. Also, detailed assessments (as was done with the $1000 \mathrm{~Hz}$ phenomena) to understand and track through time the major phenomena suggested in this work are still future activities.

Another effort that is strongly suggested by this work is to understand the $1000 \mathrm{~Hz}$ phenomena. This phenomena does show some promise for capturing the evolving state of the test article and understanding its source and characteristics will prove very enlightening.

There are modelling efforts underway to generate numerical models consistent with this accelerometer complement and the test article configuration. These models would also be used to enhance understanding of the test data and initiate efforts associated with Round 2 of the analysis plan.

\section{Conclusions}

The efforts described in this work demonstrate that structural dynamics information can be obtained and utilized in inflatable field and laboratory test situations. An assessment of the strap pops or impulse events has been performed using a normalization to allow the magnitude to be compared spatially across the sensors and temporally across the test. This assessment found four classes of impulse events. While all of the classes are likely rooted in the same basic phenomena, an understanding of the differences will shed light on the characteristics of inflatable space structures. The impulse events seen in this test have been challenging to overcome, but techniques based on the transfer function approach have been shown to offset the effects of these events. There is still much work to be done to understand the modal data from this test. However, the modal content appears to contain potential noise phenomena, constant modes, pressure sensitive dynamics, and time dependent frequencies. There are indications from the assessment of the $1000 \mathrm{~Hz}$ phenomena that such data analyses will add insight into the evolution of the test article. Specifically the $1000 \mathrm{~Hz}$ mode continued to possess an increasing frequency trend even with a constant pressure. Also the amplitude of the phenomena increased significantly as failure approached. An understanding of this phenomena and its characteristics may lead to better metrics to track damage and health in similar structures.

\section{Acknowledgements}

There were a significant group of individuals who have made this work possible. The test directors, conductors, and facility team that designed, enabled, and carried out the test were essential to the ability to acquire this data. And that acknowledgement goes out to the individuals that were responsible for cleaning up after the test as well. Gerard Valle was the project lead that supported and enabled the addition of the accelerometers to this test. Juan Carlos Lopez, Dorothy Jorgensen, and Amanda Smith assisted in the modal test. Doug Litteken performed the strap test. Matt Hernandez, John-Michael Tucker, and Juan Carlos Lopez worked the final test preparations and accelerometer

data collection. Jeffery Bell played a lead role in developing the MIDOS software. Matt Hernandez, John-Michael Tucker, and Jeff Bell performed the quick-look data analysis. Amanda Smith assisted in the clean-up after the test.

American Institute of Aeronautics and Astronautics 


\section{References}

${ }^{1}$ G. James, et. al., "Operational Analysis in the Launch Environment”, Proceedings of the $30^{\text {th }}$ International Modal Analysis Conference, Jacksonville, FL, January 30 - February 2, 2012.

${ }^{2}$ M. Selig, et. al., "Creep Burst Testing of a Woven Inflatable Module", Proceedings of the $56^{\text {th }}$ AIAA/ASME/ASCE/AHS/ASC Structures, Structural Dynamics, and Materials Conference, January 5-9, 2015.

${ }^{3}$ M. Grygier, "Modal Test Technology as Non-Destructive Evaluation of Space Shuttle Structures," NASA CP-3263, 1994, pp. 329-334, NASA-JSC, Houston, TX, January, 1987.

${ }^{4}$ T. Cao, et. al., "Identification of Ritz Vectors from Ambient Test Data," Proceedings of the $16^{\text {th }}$ International Modal Analysis Conference, Santa Barbara, CA, February 2-5, 1998.

${ }^{5}$ G. James, et. al., "Reconstruction of the Space Shuttle Roll-Out Forcing Function", Proceedings of the $25^{\text {th }}$ International Modal Analysis Conference, Orlando, FL, February 19-23, 2007.

${ }^{6} \mathrm{H}$. Herlufsen, "Modal Analysis using Multi-Reference and Multi-Input Multipe-Output Techniques", Application Note BO0505-12, Brüel\&Kjær, Denmark, February, 2012.

${ }^{7}$ A. Phillips, et, al., "A Comparison of MIMO-FRF Excitation/Averaging Techniques on Heavily and Lightly Damped Structures", Proceedings of the 17 $7^{\text {th }}$ International Modal Analysis Conference, Orlando, FL, February 8-11, 1999.

${ }^{8}$ J.-N. Juang, et, al., "Identification of Observer/Kalman Filter Markov Parameters: Theory and Experiments", Journal of Guidance, Control, and Dynamics, Vol. 16, No. 2, March-April 1993.

${ }^{9}$ J.-N. Juang, et, al., "System/Observer/Controller Identification Toolbox", NASA Technical Memorandum \#107566, NASA Langley Research Center, VA, February, 1992.

${ }^{10}$ J.-N. Juang, et, al., “An Eigensystem Realization Algorithm Using Data Correlation (ERA/DC) for Modal Parameter Identification", Control Theory and Advanced Technology, Vol. 4, No. 1, January, 1988.

${ }^{11}$ R. Pappa, et, al., "Consistent-Mode Indicator for the Eigensystem Realization Algorithm", Journal of Guidance, Control, and Dynamics, Vol. 16, No. 5, September-October, 1993.

${ }^{12} \mathrm{G}$. James, et, al., "Development of a Coupled Approach for Structural Damage Detection with Incomplete Measurements", AIAA Journal, Vol. 36, No. 12, December, 1998.

American Institute of Aeronautics and Astronautics 